COMMUNICATIONS IN

ANALYSIS AND GEOMETRY

Volume 11, Number 1, 49-83, 2003

\title{
On the Well-posedness of the Wave Map Problem in High Dimensions
}

\author{
Andrea Nahmod, Atanas Stefanov and Karen Uhlenbeck
}

\begin{abstract}
We construct a gauge theoretic change of variables for the wave map from $\mathbb{R} \times \mathbb{R}^{n}$ into a compact group or Riemannian symmetric space, prove a new multiplication theorem for mixed LebesgueBesov spaces, and show the global well-posedness of a modified wave map equation - $n \geq 4$ - for small critical initial data. We obtain global existence and uniqueness for the Cauchy problem of wave maps into compact Lie groups and symmetric spaces with small critical initial data and $n \geq 4$.
\end{abstract}

\section{Introduction.}

The wave map equation between two Riemannian manifolds- the wave equation version of the evolution equations which are derived from the same geometric considerations as the harmonic map equation between two Riemannian manifolds- has been studied by a number of mathematicians in the last decade. The work of Klainerman and Machedon [8] [9] [10] and of Klainerman and Selberg [12] focus on the study of the general Cauchy problem in any dimensions bigger or equal then two for regular data and provide the almost optimal local well-posedness. In the difficult case of two dimensions, some of the early results include those of Christodoulou and Tahvildar-Zadeh [3] who studied the regularity of spherically symmetric wave maps assuming a convexity condition in the target manifold. Shatah and Tahvildar-Zadeh [17] [18] studied the optimal regularity of equivariant wave maps into two-dimensional rotationally symmetric and geodesically convex Riemannian manifolds. The study of the general wave map problem incorporated methods that exploited the null-form structure of the wave map system such as that of M. Grillakis [4] [5] as well as the geometric structure

${ }^{1}$ The first author was partially supported by NSF grant DMS 9971159. The first and third authors acknowledge the support and hospitality of IAS at Princeton (May 2001), where part of this research was carried out. The third author would like to thank Montana State University for their hospitality as well. 
of the equation as done by M. Struwe [19] [20] [21]. Keel and Tao studied the one (spatial) dimensional case in [7]. For a more complete presentation of the developments in this subject we refer the interested reader to the excellent book by Shatah and Struwe [16].

More recently, the pivotal work of Tataru [24], [25], followed by that of Tao [22] [23] have introduced new techniques which allow one to treat the Cauchy problem with critical data. Their methods rely and further develop important ideas from harmonic analysis such as adapted frequency decompositions and Littlewood-Paley theory; and in Tao's case are used in conjunction with gauge theoretic geometric methods. Although the general theory for these equations is far from complete, the field seems at present very promising.

In [22], Tao established the global regularity for wave maps from $\mathbb{R} \times$ $\mathbb{R}^{n}$ into the sphere $\mathbb{S}^{m}$ when $n \geq 5$. Similar results to those of Tao were obtained by Klainerman and Rodnianski [11] for target manifolds that admit a bounded parallelizable structure.

In this paper we are interested in revisiting this work. We study the Cauchy problem for wave maps from $\mathbb{R} \times \mathbb{R}^{n}$ into a (compact) Lie group (or Riemannian symmetric spaces) when $n \geq 4$ and establish global exisitence and uniqueness provided the Cauchy initial data are small in the critical norm. Similar results were obtained by Shatah and Struwe at roughly the same time when the target is any complete Riemannian manifold with bounded curvature.

Our method combines both delicate techniques from harmonic analysis with fairly standard global gauge theoretic geometric methods. Both our work and that of Shatah-Struwe [15] use the same gauge change; the analytic approach however, is significatly different as Shatah-Struwe base their results on Lorentz spaces and we use Besov spaces. Besov spaces are contained in Lorentz spaces -for appropriate indices- (c.f. [16] for example). Lorentz spaces seem to be better behaved under coordinate transformations.

It is interesting to note that in none of the works above is possible to obtain (strong) well posedness at the critical level for the wave map itself. In other words even though one indeed has well posedness for the gauged map; there are no estimates available on differences for the original wave map itself and one cannot obtain any continuous dependence of the map on the data in the coordinate setting. It thus seems reasonable to think that the notion of wellposedness is not appropriate for this type of geometric equations at the critical level. The problem stems in that well posedness is not a gauge invariant notion; it is not even necessarily true that uniqueness in one coordinate system implies uniqueness in another directly. 
The plan for this paper is as follows. In section 1 we describe the geometry which translates wave maps into compact groups and Riemannian symmetric spaces to a gauged equation - the gauged wave map (GWM). This equation is overdetermined and we give a modified version (MWM). Section 2 contains the basic estimates for our theory, which involve multiplication theorems in Lebesgue-Besov spaces. Proposition 2.12 is the key estimate. This is the tool which contributes to handling the notoriously difficult first derivative non-linearity of the wave equation

$$
\square u+a \cdot d u=0 .
$$

We obtain our results using the quadratic structure of the definition of $a$ in terms of $b \quad$ (which is linear in $d u$ )

$$
\Delta a+\operatorname{div}([b, b]+[a, a])=0 .
$$

This estimate is the subject of section 3. Section 4 contains the proof of the global well-posedness of the modified wave map equation (MWM) for small initial data in the scale invariant norm $\dot{H}^{n / 2}$. In section 4 we briefly outline the translation back to the original wave map coordinates. Our main result is the existence and uniqueness of global wave maps into compact Lie groups and symmetric spaces for small initial data in $\dot{H}^{n / 2} \times \dot{H}^{n / 2-1}$ for $n \geq 4$.

There are small difficulties in handling the case of non-compact symmetric spaces. The natural isometric embeddings are into spaces with indefinite metrics. For the standard methods on density theorems and coordinate changes to apply, it is necessary to know the existence of a Nash embedding into an Euclidean space with bounded geometry.

Our results extend the results of Tao and Tataru for $M=\mathbb{S}^{m}$. The Shatah-Struwe methods using Lorentz spaces are stronger since they obtain estimates for solutions with variable curvature. ${ }^{1}$ (On the surface, our difficulties with non-compact targets have somehow been circumvented in their work [15]).

We have stated the results in sections 1 and 2 in great generality in the hopes that they may be applicable to other non-linear wave equations. The Main Multiplication theorem is the principal tool needed in estimating the non-linearity term in Theorem (2.13).

The authors particularly thank both J. Shatah and T. Tao for their generous sharing of information and suggestions in a field relatively new to us, as well as for their enthusiastic support.

${ }^{1} \mathrm{~F}$. Planchon has recently pointed out that actually, multiplication theorems for Besov spaces and $L^{\infty}$ are sufficient to include variable curvature in our proof of the main theorem. 


\section{Formulation of the problem and gauge choices.}

We regard the wave map equation as an equation given through covariant derivatives. These arise as follows:

$$
s: \mathbb{R} \times \mathbb{R}^{n} \rightarrow M
$$

where $M$ is an arbitrary Riemannian manifold and

$$
d s: \mathcal{T}\left(\mathbb{R} \times \mathbb{R}^{n}\right) \rightarrow \mathcal{T} M
$$

where $\mathcal{T}\left(\mathbb{R} \times \mathbb{R}^{n}\right)=\left(\mathbb{R} \times \mathbb{R}^{n}\right) \times\left(\mathbb{R} \oplus \mathbb{R}^{n}\right)$.

Let $s^{*} \nabla$ be the pullback of the Levi-Civita connection on $M$ to $s^{*} \mathcal{T} M$ via the map $s$. Then, in coordinate free notation, the wave map equation is

$$
s^{*} \nabla_{0} \frac{\partial s}{\partial t}-\sum_{j=1}^{n} s^{*} \nabla_{j} \frac{\partial s}{\partial x^{j}}=0 .
$$

Since the Levi-Civita connection on $M$ is torsion free,

$$
s^{*} \nabla_{j} \frac{\partial s}{\partial x^{k}}=s^{*} \nabla_{k} \frac{\partial s}{\partial x^{j}},
$$

for $j=0,1, \ldots, n, k=1, \ldots, n$ where we have set $t=x^{0}$.

We assume the map $s$ is topologically trivial which is usually implied by the later curvature bounds. (The wave map fixes spatial infinity so topologically, $\left.s: \mathbb{R}^{n} \cup\{\infty\} \rightarrow M\right)$. Hence, $s^{*} \mathcal{T} M$ is the trivial bundle $\left(\mathbb{R}^{1} \times \mathbb{R}^{n}\right) \times \mathbb{R}^{m}$. We also have control on the curvature of $s^{*} \nabla$ via the equation

$$
\left[s^{*} \nabla_{j}, s^{*} \nabla_{k}\right]=R(s)\left(\frac{\partial s}{\partial x^{j}}, \frac{\partial s}{\partial x^{k}}\right) .
$$

Our first theorem asserts that under smallness assumptions on $s \in$ $L_{t}^{\infty} \dot{W}_{x}^{1, n / 2}$, there is a unique choice of coordinates for $s^{*} \mathcal{T} M$. Given a smooth map $s$ with sufficient decay in asymptotics (to a point) at infinity, the initial coordinates can be found by a partition of unity. The theorem we need is stated in a more general framework, as we hope to find applications for this theorem in gauge theory.

Theorem 1.1. Let $d+A$ be a smooth connection with compact structure groups $G$ over $\mathbb{R} \times \mathbb{R}^{n}$ or $I \times \mathbb{R}^{n}$. Assume $A \sim 0$ at spatial infinity and let $F_{A}=d A+[A, A]$ be the space-time curvature. Then there exists a positive constant $\epsilon=\epsilon(n, G)$ such that if the mixed space-time Lebesgue norm

$$
\left\|F_{A}\right\|_{L_{t}^{\infty} L_{x}^{n / 2}}<\epsilon
$$


then, there exits a unique smooth gauge change $g, g \sim I$ at spatial infinity, such that if $\tilde{A}=g A g^{-1}-d g g^{-1}$ we have,

(1) $\|\tilde{A}\|_{L_{t}^{\infty} \dot{W}_{x}^{1, n / 2}} \leq c(n, G)\left\|F_{A}\right\|_{L_{t}^{\infty} L_{x}^{n / 2}}$

(2) $\sum_{j=1}^{n} \frac{\partial}{\partial x^{j}} \tilde{A}_{j}=0$

Proof. The method of proof follows the method used by the third author in [26]. We omit the dependence of constants on $G$ in the following proof. First, we fix each time slice $t=t_{0}$. The methods of [26] show that in every ball $B_{N}=\{x:|x| \leq N\}$ there exists a gauge change $g_{N}$ such that the spatial part of the connection $A_{N}=g_{N} A g_{N}^{-1}-d g_{N} g_{N}^{-1}$ satisfies

$$
\left\|A_{N}\right\|_{t, \dot{W}_{x}^{1, n / 2}\left(B_{N}\right)} \leq c(n)\left\|F_{A}\right\|_{t, L_{x}{ }^{n / 2}\left(B_{N}\right)} .
$$

By taking $N \rightarrow \infty, g_{N} \rightarrow g, A_{N} \rightarrow \tilde{A}$, and we obtain a solution

$$
\tilde{A}=g A g^{-1}-d g g^{-1}, \quad \sum_{j=1}^{n} \frac{\partial}{\partial x^{j}} \tilde{A}=0
$$

on each time slice $\left(t, \mathbb{R}^{n}\right)$ which satisfies on all $\mathbb{R}^{n}$,

$$
\|\tilde{A}\|_{t, \dot{W}_{x}^{1, n / 2}} \leq c(n)\left\|F_{A}\right\|_{t, L_{x}^{n / 2}} .
$$

Since $A$ is asymptotic to 0 at infinity, $d g$ is as well, and we may choose $g \sim I$ at spatial infinity as well. Let $g=\exp (u)$. We fix a time slice and then differentiate in $t$. Namely, if

$$
\sum_{j=1}^{n} \frac{\partial}{\partial x^{j}} \exp (u) \tilde{A}_{j} \exp (-u)-\frac{\partial}{\partial x^{j}} \exp (u) \exp (-u)=0
$$

is the equation at the time slice $t_{0}$, the derivative at $t=t_{0}$ is

$$
L_{\tilde{A}} u=\Delta u+d *[u, \tilde{A}]=\Delta u+[d u, \tilde{A}] .
$$

Here we use the fact that $\frac{\partial}{\partial x^{j}} \tilde{A}_{j}=0$ at $t=t_{0}$. Examine the properties of this linear map -which is the derivative-

$$
L_{\tilde{A}}: \dot{L}^{2, n / 2} \rightarrow L^{n / 2}, \quad L_{\tilde{A}}=\Delta+\text { lower order terms. }
$$


We have

$$
\begin{aligned}
\|[d u, \tilde{A}]\|_{L^{n / 2}} & \leq 2\|d u\|_{L^{n}}\|\tilde{A}\|_{L^{n}} \\
& \leq c(n)\|u\|_{\dot{W}^{2, n / 2}}\|A\|_{\dot{W}^{1, n / 2}} \\
& \leq c(n) \epsilon\|u\|_{\dot{W}^{2, n / 2}} .
\end{aligned}
$$

Choose $\epsilon$ so that $c(n) \epsilon<1 / 2$; we have that the lower order term is small enough for $L_{\tilde{A}}$ to be invertible. The precise estimate is for $u=\frac{\partial g}{\partial t} g^{-1}$ where

$$
L_{\tilde{A}} u+\sum_{j=1}^{n} \frac{\partial}{\partial x^{j}}\left(g \frac{\partial A}{\partial t} g^{-1}\right)=0 .
$$

Note that an estimate on $\frac{\partial g}{\partial t}$ is available by the general methods we have been using.

To obtain an estimate on the time component $\tilde{A}_{0}=g A_{0} g^{-1}-\frac{\partial g}{\partial t} g^{-1}$, note that

$$
d \tilde{A}_{0}-\frac{\partial \tilde{A}}{\partial t}+\left[\tilde{A}, A_{0}\right]=\left(F_{A}\right)_{(\text {space,time })} .
$$

Since $d * \tilde{A}=0$, we have

$$
\Delta \tilde{A}_{0}+\frac{\partial}{\partial x^{j}}\left[\tilde{A}_{j}, A_{0}\right]=\sum_{j=1}^{n} \frac{\partial}{\partial x^{j}}\left(F_{\tilde{A}}\right)_{j, 0} .
$$

Let $\mu_{j}=\Delta^{-1 / 2} \frac{\partial}{\partial x^{j}}$. Then

$$
\begin{aligned}
\left\|\tilde{A}_{0}\right\|_{\dot{W}^{1, n / 2}} & =\left\|\Delta^{1 / 2} \tilde{A}_{0}\right\|_{L^{n / 2}} \\
& \leq\left\|\sum_{j=1}^{n} \mu_{j}\left[\tilde{A}_{j}, \tilde{A}_{0}\right]_{L^{n / 2}}+\right\| \sum_{j=1}^{n} \mu_{j}\left(F_{\tilde{A}}\right)_{j, 0} \|_{L^{n / 2}} \\
& \leq c(n)\left(\|\tilde{A}\|_{L^{n}}\left\|\tilde{A}_{0}\right\|_{L^{n}}+\left\|F_{\tilde{A}}\right\|_{L^{n / 2}}\right) \\
& \leq c(n)\left(\epsilon\left\|\tilde{A}_{0}\right\|_{L^{n}}+\left\|F_{A}\right\|_{L^{n / 2}}\right) .
\end{aligned}
$$

Again, under the assumption $c(n) \epsilon<1 / 2$, we have

$$
\left\|\tilde{A}_{0}\right\|_{\dot{W}^{1, n / 2}} \leq 2 c(n)\left\|F_{A}\right\|_{L^{n / 2}}
$$

as claimed.

Corollary 1.2. Theorem (1.1) remains true if $A \in L_{t}^{\infty} \dot{W}_{x}^{1, n / 2}$ and $F_{A} \in$ $L_{t}^{\infty} L_{x}^{n / 2}$ 
Proof. Approximate $A$ by smooth connections $A_{\alpha} \rightarrow A$ in $\dot{W}^{1, n / 2}$. For each $\alpha$, construct $g_{\alpha}$ as in the theorem and $A \alpha=g_{\alpha} A_{\alpha} g_{\alpha}^{-1}-\hat{d} g_{\alpha} g_{\alpha}^{-1}$ a spacetime connection 1 -form which satisfies the estimates. We have denoted by $\hat{d}=\left(\frac{\partial}{\partial t}, d\right)$ the full derivative. Then,

$$
\begin{aligned}
\left\|\hat{d} g_{\alpha}\right\|_{L^{n}} & =\left\|\hat{d} g_{\alpha} g_{\alpha}^{-1}\right\|_{L^{n}} \\
& \leq\left\|\tilde{A}_{\alpha}\right\|_{L^{n}}+\left\|g_{\alpha} A_{\alpha} g_{\alpha}^{-1}\right\|_{L^{n}} \\
& \leq\left\|\tilde{A}_{\alpha}\right\|_{L^{n}}+\left\|A_{\alpha}\right\|_{L^{n}} .
\end{aligned}
$$

Hence $\|\hat{d} g\|_{L^{n}}$ is bounded on each time-slice. To complete the estimate note that

$$
\begin{aligned}
\left(\frac{\partial g}{\partial x^{j}}\right)_{\alpha} & =\left(\tilde{A}_{\alpha}\right)_{j} g_{\alpha}-g_{\alpha}\left(A_{\alpha}\right)_{j} \\
d\left(\frac{\partial g}{\partial x^{j}}\right)_{\alpha} & =d\left(\tilde{A}_{\alpha}\right)_{j} g_{\alpha}-g_{\alpha} d\left(A_{\alpha}\right)_{j}+\left(\tilde{A}_{\alpha}\right)_{j} d g_{\alpha}-d g_{\alpha}\left(A_{\alpha}\right)_{j} .
\end{aligned}
$$

Then again, on each time slice

$$
\left\|\hat{d} g_{\alpha}\right\|_{W^{1, n / 2}} \leq\left\|\tilde{A}_{\alpha}\right\|_{W^{1, n / 2}}+\left\|A_{\alpha}\right\|_{W^{1, n / 2}}+\left(\left\|\tilde{A}_{\alpha}\right\|_{L^{n}}+\left\|A_{\alpha}\right\|_{L^{n}}\right)\left\|d g_{\alpha}\right\|_{L^{n}}
$$

is also bounded.

In each time slice, we have subsequences which converge to weak limits $g_{\alpha^{\prime}} \rightarrow g$ in $\dot{W}^{2, n / 2}$. However, the weak limit is unique. Suppose not. Then,

$$
\begin{array}{rlrl}
g_{\alpha^{\prime}} \rightarrow g, & g_{\alpha^{\prime \prime}} & \rightarrow h g ; \\
\tilde{A}=g A g^{-1}-d g g^{-1} & \text { and } \quad \quad \tilde{\tilde{A}}=h \tilde{A} h^{-1}-d h h^{-1} .
\end{array}
$$

Both $\tilde{A}$ and $\tilde{\tilde{A}}$ satisfy the time-slice estimate

$$
\|\tilde{A}\|_{\dot{W}^{1, n / 2}}+\|\tilde{\tilde{A}}\|_{\dot{W}^{1, n / 2}} \leq 2 \epsilon
$$

as well as

$$
\sum_{j=1}^{n} \frac{\partial}{\partial x^{j}} \tilde{A}_{j}=\sum_{j=1}^{n} \frac{\partial}{\partial x^{j}} \tilde{\tilde{A}}_{j}=0 .
$$

But $d h=h \tilde{A}-\tilde{\tilde{A}} h$ and $\Delta h=(d h \tilde{A})-(\tilde{\tilde{A}} d h)$. If we let $k_{n}$ be the appropriate Sobolev constant,

$$
\begin{aligned}
\|d h\|_{L^{n}} & \leq c(n)\|\Delta h\|_{L^{n / 2}} \\
& \leq c(n) k_{n}\|d h\|_{L^{n}}\left(\|\tilde{A}\|_{L^{n}}+\|\tilde{\tilde{A}}\|_{L^{n}}\right) \\
& \leq c(n) k_{n} \epsilon\|d h\|_{L^{n}} .
\end{aligned}
$$


If $2 c(n) k_{n} \epsilon<1$ we have that $d h=0$. Since $h \sim I$ at infinity, $h \equiv I$. Thus the weak limit is unique. Hence, $g$ is unique and

$$
\hat{d} g_{\alpha} \rightarrow \hat{d} g \text { in } \dot{W}^{1, n / 2}, \quad \tilde{A}_{\alpha} \rightarrow \tilde{A} \quad \text { in } \dot{W}^{1, n / 2}
$$

and

$$
\|\tilde{A}\|_{L_{t}^{\infty} \dot{W}_{x}^{1, n / 2}} \leq c(n)\left\|F_{A}\right\|_{L_{t}^{\infty} L_{x}^{n / 2}}
$$

as claimed.

Corollary 1.3. Let $s: \mathbb{R}^{1} \times \mathbb{R}^{n} \rightarrow M$ be an arbitrary map. Suppose the curvature $R(M)$ is bounded by $K$. There exists $0<\delta=\delta(n, K)$ such that if $s: \mathbb{R}^{n} \cup\{\infty\} \rightarrow N$ is topologically trivial, and

$$
\|d s\|_{L_{t}^{\infty} L_{x}^{n}} \leq \delta
$$

then there exists a unique frame in $s^{*} \mathcal{T} M$ such that the hypotheses of (1.1)

- the main gauge-fixing theorem- are satisfied.

Proof. Since

$$
\left(F_{s^{*} \nabla}\right)_{k j}=R(s)\left(\frac{\partial s}{\partial x^{j}}, \frac{\partial s}{\partial x^{k}}\right),
$$

on time slices we have the estimate

$$
\left\|F_{s^{*} \nabla}\right\|_{L^{n / 2}} \leq K\|d s\|_{L^{n}}^{2} .
$$

The desired conclusion follows by choosing $\delta>0$ such that $K \delta<\epsilon$ where $0<\epsilon=\epsilon(n, M)$ is as in Theorem (1.1).

Next we give a coordinate invariant description of the wave equations. Let

$$
D=s^{*} \nabla=d+a,
$$

where the curvature of $d$ is

$$
F_{A}=(R \circ s)(d s, d s) .
$$

The term $(R \circ s)$ is not explicit unless one is working on a Lie group or symmetric space.

Let $b=d s$. Then the equations themselves are written

$$
D_{0} b_{0}-\sum_{j=1}^{n} D_{j} b_{j}=0
$$


Because the Levi-Civita connection on $M$ has no torsion, we find

$$
D_{k} b_{j}=D_{j} b_{k}, \quad k=0,1, \ldots, n, j=1,2, \ldots, n .
$$

This is a non-linear first order hyperbolic system. It may be that the correct method is to analyze this directly. In keeping with the present standard methods, we convert it to a single equation using Hodge theory (see also [3]). In what follows we will denote by $d$ the exterior differentiation operator and by $d *=d i v_{(\text {space,time })}$ its dual, the space-time divergence operator computed using the Lorentz metric.

Theorem 1.4. Let $b=d \phi+d * \psi$. Then the wave map equations can be rewritten as

$$
\begin{array}{ll}
\text { (a) } & \square \phi+(a, b)=0 \\
\text { (b) } & \square \psi+a \wedge b=0 \\
\text { (c) } & b=d \phi+d * \psi \\
\text { (d) } & d a+[a, a]=R(x)[b, b] \\
\text { (e) } & \sum_{j=1}^{n} \frac{\partial}{\partial x^{j}} a_{j}=0 .
\end{array}
$$

Here $R(x)$ is the Riemannian curvature of $M$ evaluated at $s(x)$.

The initial data on $\phi$ and $\psi$ can be taken to be

$$
\begin{array}{lc}
\phi(0, x)=0, & \psi(0, x)=0 \\
\frac{\partial \phi}{\partial t}(0, x)=b_{0}(0, x) & \frac{\partial \psi}{\partial t}{ }_{0, j}(0, x)=b_{j}(0, x) \\
\frac{\partial}{\partial t} \psi_{j, k}(0, x)=0 & j, k \neq 0
\end{array}
$$

Proof. Let $b=\square q$ with $q(0, x)=\frac{\partial q}{\partial t}(0, x)=0$ where $\square=d d *+d * d$.

Let

$$
\phi=d * q=\frac{\partial}{\partial t} b_{0}-\sum_{j=1}^{n} \frac{\partial}{\partial x^{j}} b_{j}
$$

and

$$
\psi=d q=\frac{\partial}{\partial t} b_{j}-\frac{\partial}{\partial x^{j}} b_{0}, \frac{\partial}{\partial x^{k}} b_{j}-\frac{\partial}{\partial x^{j}} b_{k}
$$

Hence

$$
\square \phi=d * b \quad \text { and } \quad \square \psi=d \wedge b
$$


So, $b=d \phi+\operatorname{div}_{(\text {space,time })} \psi$. Note $d \psi=0$ automatically.

The initial data clearly consists of $\phi(0, x)=0, \psi(0, x)=0$. Hence,

$$
b_{0}=\frac{\partial}{\partial t} \phi-\sum_{j=1}^{n} \frac{\partial}{\partial x^{j}} \psi_{j, 0}, \quad \text { so } \quad b_{0}(0, x)=\frac{\partial}{\partial t} \phi(0, x) .
$$

Likewise,

$$
b_{j}=\frac{\partial}{\partial x^{j}} \phi-\frac{\partial}{\partial t} \psi_{0, j}+\sum_{k=1}^{n} \frac{\partial}{\partial x^{k}} \psi_{k, j}, \quad \text { so } \quad b_{j}(0, x)=\frac{\partial}{\partial t} \psi_{0, j}(0, x) .
$$

Note also that

$$
\frac{\partial}{\partial t} \psi_{j, k}=\frac{\partial}{\partial x^{j}} \psi_{0, k}+\frac{\partial}{\partial x^{k}} \psi_{j, 0}, \quad \text { so } \quad \frac{\partial}{\partial t} \psi_{j, k}(0, x)=0 .
$$

The last equation (d) of (1.4) is not determined by the rest of the data since the curvature depends on the original map (and gauge change). No general formula is available. This would not preclude a priori estimates. However, the estimates for our global existence and uniqueness theorem for wave maps are done in Besov spaces (which here prove inferior to the Lorentz spaces). The equation

$$
d a+[a, a]=R(x)[b, b]
$$

however behaves 'badly' (for bounded $R(x)$ ) in this context. Hence we must restrict the manifold $M$ to a group or a Riemannian symmetric space.

Theorem 1.5. If $M=G$ or $M=H / G$ where $G$ is a compact Lie group, then the equation (d) in Theorem (1.4) can be replaced by the equation

$$
\text { (d)' } \quad d a+[a, a]+[b, b]=0 .
$$

Moreover, the original map $s: \mathbb{R}^{1} \times \mathbb{R}^{n} \rightarrow G$ (or $H / G$ ) can easily be reconstructed from the fact that $d+a+b$ and $d+a-b$ are flat connections. Let

$$
(d+a+b) g^{+}=0 \quad \text { and } \quad(d+a-b) g^{-}=0 .
$$

The original map is $g=g^{+} \cdot g^{-}$.

Proof. The computations for a Lie group are straightforward if we remember that $\mathcal{T}^{*} G=\mathfrak{G}$, the Lie algebra of $\mathrm{G}$, that $[\cdot, \cdot]$ generates curvature, and that the structure group is a specialization of the orthogonal group. The 
symmetric space case is best understood by regarding $M$ as an $A d$ orbit in the possibly non-compact group $H$. That is,

$$
M=A d H(\hat{i})
$$

and $G$ is the (compact) isotropy subgroup of $\hat{i}$. For $\mathbb{H}^{m}, H$ is the Lorentz group $\mathcal{O}(1, m)$ and $G$ is the Euclidean group $\mathcal{O}(m)$. Choose $\hat{i}=\operatorname{diag}(1,-1,-1, \ldots,-1)$. Then by construction $b$ will always lie in the off-diagonal vectors

$$
b_{j}=\left[\begin{array}{cccc}
0 & \cdot & v_{j} & \cdot \\
\cdot & 0 & \ldots & 0 \\
-v_{j}^{*} & 0 & \ldots & 0 \\
\cdot & 0 & \ldots & 0
\end{array}\right],
$$

and the compact structure group $\mathcal{O}(m)$ is represented on the diagonal. The construction cannot work for non-compact Lie groups $H$ such as $\mathcal{O}(1, m)$ since the do not have bi-invariant Riemannian metrics.

Corollary 1.6. Suppose $M=G$ or $M=H / G$. Then a subset of the gauged wave map equations (a)-(e) (GWM) has a structure of a non-linear wave system of integral differential operators.

(a) $\square \phi+(a, b)=0$

(b) $\square \psi+a \wedge b=0$

(c) $\quad b=d \phi+d * \psi$

(d) $\quad \Delta a_{j}+\sum_{k=1}^{n} \frac{\partial}{\partial x^{k}}\left[a_{k}, a_{j}\right]+\frac{\partial}{\partial x^{k}}\left[b_{k}, b_{j}\right]=0, \quad j=0,1, \ldots n$.

Proof. The wave equation structure of the system in $\phi$ ad $\psi$ is clear, and $b$ is a (linear) first order derivative of $\phi$ and $\psi$ (note that the initial data for $\phi$ and $\psi$ have been worked out as coupled to that of $b$.)

$$
\begin{aligned}
& \phi(0, x)=\psi(0, x)=0 \\
& \frac{\partial \phi}{\partial t}(0, x)=b_{0}(0, x) \\
& \frac{\partial \psi_{j, 0}}{\partial t}(0, x)=b_{j}(0, x) \\
& \frac{\partial \psi_{j, k}}{\partial t}(0, x)=0 . \\
&
\end{aligned}
$$

To obtain the last equation, note that

$$
\frac{\partial}{\partial x^{k}} a_{j}-\frac{\partial}{\partial x^{j}} a_{k}+\left[a_{j}, a_{k}\right]+\left[b_{j}, b_{k}\right]=0
$$


for $k=1,2, \ldots, n, \quad j=0,1, \ldots, n$ and $\frac{\partial}{\partial x^{0}}=\frac{\partial}{\partial t}$. Since $\sum_{k=1}^{n} \frac{\partial}{\partial x^{k}} a_{k}=$ 0 , we obtain our new equation by taking the divergence. We call this system the modified wave map (MWM). By indirect arguments, it is clear that if $(\phi, \psi, b, a)$ have initial data which satisfy appropriate constraints, the evolution, at least in the smooth case, will actually be data coming from a wave map. The direct argument is not available to us however.

\section{Definitions and Product Estimates.}

In this section we set out definitions, notations and basic estimates that will be used throughout the paper. We shall frequently use the notation $A \lesssim B$ to mean $A \leq$ const. $B$ for some positive constant const. which is allowed to vary from line to line but does not depend on any of the relevant parameters in the estimates.

We begin reviewing some Littlewood-Paley theory. Let $\phi(t, x)$ be a function on $\mathbb{R} \times \mathbb{R}^{n}$, we define the spatial Fourier transform $\hat{\phi}(t, \xi)$ by

$$
\hat{\phi}(t, x)=\int_{\mathbb{R}^{n}} e^{-2 \pi i x \cdot \xi} \phi(t, x) d x
$$

We define now the usual Littlewood-Paley projection operators $P_{k}$ and $Q_{k}$. To that effect, let $m(\xi)$ be a non-negative radial bump function supported on the ball $|\xi| \leq 2$ and equal to 1 on the ball $|\xi| \leq 1$. Then for each integer $k$ we define $P_{k}(\phi)$ the projection onto the frequency ball $|\xi| \lesssim 2^{k}$ by

$$
\widehat{P_{k}(\phi)}(\xi):=m\left(2^{-k} \xi\right) \hat{\phi}(t, \xi) .
$$

Note that $P_{k} \rightarrow 0$ in $L^{2}$ as $k \rightarrow-\infty$ while $P_{k} \rightarrow I$ in $L^{2}$ as $k \rightarrow \infty$.

The operator $Q_{k}$ is the projection onto the frequency annulus $|\xi| \sim 2^{k}$ given by the formula,

$$
Q_{k}:=P_{k}-P_{k-1} .
$$

We note that if we let $\psi(\xi):=m(\xi)-m(2 \xi)$, then $\psi$ is supported on the annulus $1 / 2 \leq|\xi| \leq 2$, for all $\xi \neq 0, \quad \sum_{k \in \mathbb{Z}} \psi\left(2^{-k} \xi\right) \equiv 1$, and

$$
\widehat{Q_{k}(\phi)}(t, \xi)=\psi\left(2^{-k} \xi\right) \hat{\phi}(t, \xi) .
$$

The Littlewood-Paley projections are bounded operators in all the Lebesgue spaces and commute with any constant coefficient differential operator. Finally we note that $Q_{k}$ is given by a convolution kernel whose $L^{p}$-norm equals $2^{(k n)(1-1 / p)}$ for all $1 \leq p \leq \infty$. In particular its $L^{1}$ - norm is identically 1 for all $k \in \mathbb{Z}$. 
Let $j=0$ or $j=1$ and let $k \in \mathbb{Z}$. Following [22] and also [11], we introduce $\mathcal{S}_{k}^{(-j)}\left(\mathbb{R} \times \mathbb{R}^{n}\right)$, the Strichartz space at frequency $2^{k}$ to be the space of functions whose space-time norm is given by:

$$
\|\Phi\|_{\mathcal{S}_{k}^{(-j)}}:=\sup _{q, r \in \mathcal{A}} 2^{k\left(\frac{1}{q}+\frac{n}{r}-j\right)}\left(\|\Phi\|_{L_{t}^{q} L_{x}^{r}}+2^{-k}\left\|\partial_{t} \Phi\right\|_{L_{t}^{q} L_{x}^{r}}\right),
$$

where $\mathcal{A}:=\left\{(q, r): 2 \leq q, r \leq \infty, \frac{1}{q}+\frac{n-1}{2 r} \leq \frac{n-1}{4}\right\}$ is the set of admissible Strichartz exponents. We remark that when $j=0$ the spaces above are $\dot{H}^{n / 2}$ - normalized and correspond to Tao's spaces $S_{k}$ in [22]. We also note that for each $n \geq 4$, only specific values of $(q, r)$ are needed. Finally observe that control of the $\mathcal{S}_{k}^{(-j)}$ norm gives, for example, the estimates:

$\left\|Q_{k}(\phi)\right\|_{L_{t}^{2} L_{x}^{\frac{2(n-1)}{(n-3)}}}+2^{-k}\left\|\partial_{t} Q_{k}(\phi)\right\|_{L_{t}^{2} L_{x}^{\frac{2(n-1)}{(n-3)}}} \leq 2^{k\left(j+\frac{n}{(n-1)}-\frac{(n+1)}{2}\right)}\left\|Q_{k}(\phi)\right\|_{\mathcal{S}_{k}^{(-j)}}$

$$
\left\|Q_{k}(\phi)\right\|_{L_{t}^{\infty} L_{x}^{2}}+2^{-k}\left\|\partial_{t} Q_{k}(\phi)\right\|_{L_{t}^{\infty} L_{x}^{2}} \leq 2^{k\left(j-\frac{n}{2}\right)}\left\|Q_{k}(\phi)\right\|_{\mathcal{S}_{k}^{(-j)}}
$$

$$
\left\|Q_{k}(\phi)\right\|_{L_{t}^{2} L_{x}^{\infty}}+2^{-k}\left\|\partial_{t} Q_{k}(\phi)\right\|_{L_{t}^{2} L_{x}^{\infty}} \leq 2^{k\left(j-\frac{1}{2}\right)}\left\|Q_{k}(\phi)\right\|_{\mathcal{S}_{k}^{(-j)}}
$$

Finally we state the Strichartz estimates in this framework (c.f. [22] [6] and references therein ).

Theorem 2.4 (Strichartz Estimates). Let $k$ be an integer and let $\Phi$ be any function on $\mathbb{R} \times \mathbb{R}^{n}$ with spatial Fourier support on the annulus $|\xi| \sim 2^{k}$. Then

$$
\|\Phi\|_{S_{k}^{(-j)}} \lesssim\|\Phi(0, \cdot)\|_{\dot{H}_{x}^{n / 2-j}}+\left\|\partial_{t} \Phi(0, \cdot)\right\|_{\dot{H}_{x}^{n / 2-(j+1)}}+2^{k\left(\frac{n}{2}-(j+1)\right)}\|\square \Phi\|_{L_{t}^{1} L_{x}^{2}} .
$$

Definition 2.5. Let $\mathcal{S}^{(-j)}$ be the space of functions on $\mathbb{R} \times \mathbb{R}^{n}$ whose norm is given by

$$
\|\phi\|_{\mathcal{S}^{(-j)}}:=\left(\sum_{k \in \mathbb{Z}}\left\|Q_{k}(\phi)\right\|_{S_{k}^{(-j)}}^{2}\right)^{1 / 2}
$$


Definition 2.6. A pair $(q, r)$ is said to be sharp admissible if $2 \leq q, r \leq \infty$ and

$$
\frac{1}{q}+\frac{n-1}{2 r}=\frac{n-1}{4}
$$

Remark. If $n \geq 4$ and $(q, r)$ is sharp admissible then $s=1 / q+n / r-1>0$. Also, in particular, $q \geq 2$ and $2 \leq r \leq \frac{2(n-1)}{n-3}$.

Lemma 2.7. For any $j \geq 0$ we have that

$$
\begin{aligned}
& \sup _{(q, r)-\text { admissible }} 2^{k\left(\frac{1}{q}+\frac{n}{r}-j\right)}\left\|Q_{k}(f)\right\|_{L_{t}^{q} L_{x}^{r}} \\
& =\sup _{(q, r)-\text { sharp admissible }} 2^{k\left(\frac{1}{q}+\frac{n}{r}-j\right)}\left\|Q_{k}(f)\right\|_{L_{t}^{q} L_{x}^{r} .}
\end{aligned}
$$

In other words,

$$
\begin{gathered}
\|f\|_{\mathcal{S}^{(-j)}}=\left(\sum _ { k \in \mathbb { Z } } | _ { ( q , r ) \text { -sharp admissible } } 2 ^ { k ( \frac { 1 } { q } + \frac { n } { r } - j ) } \left(\left\|Q_{k}(f)\right\|_{L_{t}^{q} L_{x}^{r}}+\right.\right. \\
\left.\left.+2^{-k}\left\|\partial_{t} Q_{k}(f)\right\|_{L_{t}^{q} L_{x}^{r}}\right)\left.\right|^{2}\right)^{\frac{1}{2}}
\end{gathered}
$$

Proof. Let $(q, r)$ be admissible but not sharp admissible and define $r_{0}$ such that $\left(q, r_{0}\right)$ is sharp admissible. Then it is clear that $r_{0}<r$. Let $s>0$ be such that

$$
\frac{1}{r}=\frac{1}{r_{0}}-\frac{\gamma}{n}
$$

By the Sobolev embedding we then have

$$
\left\|Q_{k}(f)\right\|_{L_{t}^{q} L_{x}^{r}} \leq 2^{k \gamma}\left\|Q_{k}(f)\right\|_{L_{t}^{q} L_{x}^{r_{0}}}
$$

Note that when $r=\infty, W^{n / \gamma, r_{0}} \hookrightarrow B M O$ but $\left\|Q_{k}(f)\right\|_{L^{\infty}} \sim$ $\left\|Q_{k}(f)\right\|_{B M O}$. Hence on each Littlewood-Paley piece the Sobolev embedding above holds.

Then,

$$
\begin{aligned}
2^{k\left(\frac{1}{q}+\frac{n}{r}-j\right)}\left\|Q_{k}(f)\right\|_{L_{t}^{q} L_{x}^{r}} & \leq 2^{k\left(\frac{1}{q}+\frac{n}{r}+\gamma-j\right)}\left\|Q_{k}(f)\right\|_{L_{t}^{q} L_{x}^{r_{0}}} \\
& \leq 2^{k\left(\frac{1}{q}+\frac{n}{r_{0}}-j\right)}\left\|Q_{k}(f)\right\|_{L_{t}^{q} L_{x}^{r_{0}}}
\end{aligned}
$$


and similarly for $2^{-k}\left\|\partial_{t} Q_{k}(f)\right\|_{L_{t}^{q} L_{x}^{r}}$ from where the conclusion follows.

In what follows we will denote by $|\nabla|^{-1}:=\nabla \Delta^{-1}$ be the pseudodifferential operator defined by

$$
\widehat{|\nabla|^{-1}} f(t, \xi)=\frac{1}{|\xi|} \hat{f}(t, \xi) .
$$

Definition 2.8. We denote by $\mathcal{B}_{p}$ be the Banach space of functions on $\mathbb{R} \times$ $\mathbb{R}^{n}$ whose norm is given by

$$
\|f\|_{\mathcal{B}_{p}}:=\left(\sum_{k \in \mathbb{Z}}\left\|Q_{k}(f)\right\|_{L_{t}^{1} L_{x}^{\infty}}^{p}\right)^{1 / p}
$$

for $1 \leq p<\infty$ and suitable modified with the $\ell^{\infty}$-norm when $p=\infty$

Remark. Note that it follows naturally from the embeddings $\ell^{p} \subset \ell^{q}$ that $\mathcal{B}_{p} \subset \mathcal{B}_{q}$ for $1 \leq p<q \leq \infty$.

We proceed to prove the Main Multiplication Estimate. The point of it is that it implies in particular the three multiplication estimates that will be needed later and more. It thus gives a unified framework under which to understand the action of the 'inverse gradient' $|\nabla|^{-1}$ on the space $S^{(-1)} \times$ $S^{(-1)}$. For solutions of the homogeneous wave equation, Klainerman and Tataru [13] obtained the first bilinear estimates of this type on an improved range; those can be viewed as generalizations of the well-known StrichartzPecher inequalities.

We first need some definitions. In what follows, for any $a \in \mathbb{R}$, we will denote by $a^{-}$and $a^{+}$the real number $a-1 / 100$ and $a+1 / 100$ respectively. The constant $1 / 100$ is of course arbitrary; any (fixed) small positive number will do.

Definition 2.9. Let us denote by $\mathcal{C}, \mathcal{D}, \mathcal{E}, \mathcal{G}$ the following sets of pairs $(q, p)$ where $q, p \geq 1$.

$$
\begin{gathered}
\mathcal{C}=:\left\{(q, p) \in \mathcal{A}: \frac{1}{q}+\frac{n}{p} \leq 1^{-}\right\} \\
\mathcal{D}=:\left\{(q, p): \frac{1}{2 q}+\frac{n-1}{4 p} \leq\left(\frac{n-1}{4}\right)^{-}\right\} \\
\mathcal{D}_{t}=:\left\{(q, p): q \geq 2, \frac{1}{2 q}+\frac{n-1}{4 p} \leq\left(\frac{n-1}{4}\right)^{-}\right\}
\end{gathered}
$$




$$
\mathcal{E}=:\left\{(q, p): \frac{1}{q}=\frac{1}{q_{1}}+\frac{1}{q_{2}} ; \frac{1}{p}=\frac{1}{p_{1}}+\frac{1}{p_{2}} \text { with }\left(q_{1}, p_{1}\right) \in \mathcal{A} \text { and }\left(q_{2}, p_{2}\right) \in \mathcal{C}\right\}
$$

where $\mathcal{A}$ is as above, the set of all (wave) admissible pairs. Finally, let

$$
\mathcal{G}=: \mathcal{D} \cap \mathcal{E} \quad \mathcal{G}_{t}=: \mathcal{D}_{t} \cap \mathcal{E}
$$

Remark. Note that since $\mathcal{A} \subset \mathcal{D}, \mathcal{A} \subset \mathcal{D}_{t}$, and $\mathcal{A} \subset \mathcal{E}$ we obviously have that $\mathcal{A} \subset \mathcal{G}$ and $\mathcal{A} \subset \mathcal{G}_{t}$. We will refer to the pairs in $\mathcal{G}$ as the set of 'good pairs for frequency localized wave products'.

Definition 2.10. Let $\mathcal{S}_{+}^{(-1)}$ be the space of functions on $\mathbb{R} \times \mathbb{R}^{n}$ whose norm is given by

$$
\|\phi\|_{\mathcal{S}_{+}^{(-1)}}:=\sum_{k \in \mathbb{Z}}\left\|Q_{k}(\phi)\right\|_{S_{k+}^{(-1)}}
$$

where

$$
\|\Phi\|_{\mathcal{S}_{k_{+}}^{(-1)}}:=\sup _{(q, p) \in \mathcal{G}} 2^{k\left(\frac{1}{q}+\frac{n}{p}-1\right)}\|\Phi\|_{L_{t}^{q} L_{x}^{p}}+\sup _{(q, p) \in \mathcal{G}_{t}} 2^{k\left(\frac{1}{q}+\frac{n}{p}-1\right)} 2^{-k}\left\|\partial_{t} \Phi\right\|_{L_{t}^{q} L_{x}^{p}}
$$

Lemma 2.11. We have the following embeddings

$$
\begin{array}{lll}
\mathcal{S}_{+}^{(-1)} & \hookrightarrow \mathcal{S}^{(-1)} \\
\mathcal{S}_{+}^{(-1)} & \hookrightarrow \mathcal{B}_{1} \\
\mathcal{S}_{+}^{(-1)} & \hookrightarrow L_{t}^{q} \dot{B}_{\tilde{p}, 2}^{s}
\end{array} \quad \text { for all } q \geq 2, \tilde{p} \geq 2 \text { and } s=\frac{1}{q}+\frac{n}{\tilde{p}}-1
$$

Proof. This is an easy consequence of the definition of $\mathcal{G}$, Lemma (2.7), the embeddings $\ell^{p} \subset \ell^{q}$ for $p<q$ and the fact that

$$
\|f\|_{L_{t}^{q} \dot{B}_{\tilde{p}, 2}^{s}} \lesssim\left(\sum_{k \in \mathbb{Z}} 2^{2 k s}\left\|Q_{k}(f)\right\|_{L_{t}^{q} L_{x}^{\tilde{p}}}^{2}\right)^{1 / 2}
$$

We should also note that $(q, \tilde{p}) \in \mathcal{G}$ for any $q, \tilde{p} \geq 2$.

Proposition 2.12 (Main Multiplication Estimate).

$$
|\nabla|^{-1}: \mathcal{S}^{(-1)} \times \mathcal{S}^{(-1)} \longrightarrow \mathcal{S}_{+}^{(-1)}
$$


Proof. We consider the first supremum term in the $\mathcal{S}_{+}^{(-1)}$-norm; i.e. we need to show :

$$
\sum_{l \in \mathbb{Z}} \sup _{(\tilde{q}, \tilde{p}) \in \mathcal{G}} 2^{l(1 / \tilde{q}+n / \tilde{p}-1)}\left\|Q_{l}\left(|\nabla|^{-1}(f \cdot g)\right)\right\|_{L_{t}^{\tilde{q}} L_{x}^{\tilde{p}} \lesssim\|f\|_{S^{(-1)}}\|g\|_{S^{(-1)}}}
$$

Let $f$ and $g$ be in $\mathcal{S}^{(-1)}$ and let $f_{k}=Q_{k}(f)$ and $g_{j}=Q_{j}(g)$ be their corresponding Littlewood-Paley projections. We write

$$
\begin{aligned}
|\nabla|^{-1}(f \cdot g) & =|\nabla|^{-1}\left(\sum_{k, j \in \mathbb{Z}} f_{k} \cdot g_{j}\right) \\
& =|\nabla|^{-1}\left(\sum_{k, j \in \mathbb{Z}: k \geq j} f_{k} \cdot g_{j}\right)+|\nabla|^{-1}\left(\sum_{k, j \in \mathbb{Z}: k<j} f_{k} \cdot g_{j}\right) .
\end{aligned}
$$

By symmetry of the sums, it is enough to consider only one of them. The proof for the other is identical after exchanging $k$ and $j$

Since $\operatorname{supp}\left(\widehat{f_{k} \cdot g_{k-m}}\right) \subseteq\left\{\xi:|\xi| \leq 2^{k}\right\}$ we have that $Q_{l}\left(f_{k} \cdot g_{k-m}\right) \equiv 0$ unless $k \geq l$. On the other hand, we have that $\operatorname{supp}\left(\widehat{f_{k} \cdot g_{k-m}}\right) \cap\{\xi:|\xi|<<$ $\left.2^{k-m}\right\}=\emptyset$ if $m>5$ Hence, $Q_{l}\left(f_{k} \cdot g_{k-m}\right) \equiv 0$ unless $l=k$ and $m>5$ or $m \leq 5$ and $l<k$.

Define $\tilde{Q}_{k}(f)=\sum_{k-5 \leq j \leq k+5} Q_{j}(f)$. By the above argument we conclude that it is enough to prove each of the following two estimates :

$$
\sum_{l \in \mathbb{Z}} \sup _{(\tilde{q}, \tilde{p}) \in \mathcal{D}} 2^{l(1 / \tilde{q}+n / \tilde{p}-1)} \sum_{k>l}\left\|Q_{l}\left(|\nabla|^{-1}\left(f_{k} \cdot \tilde{Q}_{k}(g)\right)\right)\right\|_{L_{t}^{\tilde{q}} L_{x}^{\tilde{p}}} \lesssim
$$

$$
\begin{gathered}
\lesssim\|f\|_{S^{(-1)}}\|g\|_{S^{(-1)}} . \\
\sum_{l \in \mathbb{Z}} \sup _{(\tilde{q}, \tilde{p}) \in \mathcal{E}} 2^{-l} 2^{l(1 / \tilde{q}+n / \tilde{p}-1)}\left\|Q_{l}\left(\sum_{m>5} f_{l} \cdot g_{l-m}\right)\right\|_{L_{t}^{\tilde{q}} L_{x}^{\tilde{p}}} \lesssim
\end{gathered}
$$

$$
\lesssim\|f\|_{S^{(-1)}}\|g\|_{S^{(-1)}}
$$

since $\mathcal{G}=\mathcal{D} \cap \mathcal{E}$.

- We consider (2.12)(i).

For each $(\tilde{q}, \tilde{p}) \in \mathcal{D}$ let $s=1 / \tilde{q}+n / \tilde{p}-1$

First note that

$$
\sum_{l \in \mathbb{Z}} \sup _{(\tilde{q}, \tilde{p}) \in \mathcal{D}} \sum_{k>l} 2^{l s}\left\|Q_{l}\left(|\nabla|^{-1}\left(f_{k} \cdot \tilde{Q}_{k}(g)\right)\right)\right\|_{L_{t}^{\tilde{q}} L_{x}^{\tilde{p}}} \leq
$$




$$
\leq \sum_{k \in \mathbb{Z}} \sum_{l<k} \sup _{(\tilde{q}, \tilde{p}) \in \mathcal{D}} 2^{l(s-1)} \| Q_{l}\left(\left(f_{k} \cdot \tilde{Q}_{k}(g)\right) \|_{L_{t}^{\tilde{q}} L_{x}^{\tilde{p}}}\right.
$$

Since $(\tilde{q}, \tilde{p}) \in \mathcal{D}$ we have that

$$
\frac{1}{2 \tilde{q}}+\frac{n-1}{4 \tilde{p}} \leq\left(\frac{n-1}{4}\right)^{-} .
$$

By the same argument used in the proof of Lemma (2.7) it is enough to take the supremum over all $(\tilde{q}, \tilde{p}) \in \mathcal{D}$ such that $\frac{1}{2 \tilde{q}}+\frac{n-1}{4 \tilde{p}}=\left(\frac{n-1}{4}\right)^{-}$. We denote this set by $\mathcal{D}^{\#}$.

Since $\tilde{p}<\infty$, let $1<p<\infty$ such that

$$
\frac{1}{\tilde{p}}<\frac{1}{p}<\frac{1}{\tilde{p}}+\frac{1}{50(n-1)} .
$$

Then we have that

$$
\frac{n-1}{4 \tilde{p}}<\frac{n-1}{4 p}<\frac{n-1}{4 \tilde{p}}+\frac{1}{200} .
$$

Let $r>1$ be such that

$$
1+\frac{1}{\tilde{p}}=\frac{1}{r}+\frac{1}{p} .
$$

By Young's inequality and Hölder's inequality we then have that

$$
\begin{aligned}
& \sum_{k \in \mathbb{Z}} \sum_{l<k} \sup _{(\tilde{q}, \tilde{p}) \in \mathcal{D}^{\#}} 2^{l(s-1)} \| Q_{l}\left(\left(f_{k} \cdot \tilde{Q}_{k}(g)\right) \|_{L_{t}^{\tilde{q}} L_{x}^{\tilde{p}}}\right. \\
& \lesssim \sum_{k \in \mathbb{Z}} \sum_{l<k} \sup _{(\tilde{q}, \tilde{p}) \in \mathcal{D}^{\#}} 2^{l(s-1)} 2^{n l(1-1 / r)}\left\|f_{k}\right\|_{L_{t}^{2 \tilde{q}} L_{x}^{2 p}\left\|\tilde{Q}_{k}(g)\right\|_{L_{t}^{2 \tilde{q}} L_{x}^{2 p} .}}
\end{aligned}
$$

But by our choice of $p$ we have that if $(\tilde{q}, \tilde{p}) \in \mathcal{D}^{\#}$ then $(2 \tilde{q}, 2 p)$ is still in $\mathcal{A}$ the set of admissible pairs. Moreover,

$$
\left(\frac{n-1}{4}\right)^{-}=\frac{1}{2 \tilde{q}}+\frac{n-1}{4 \tilde{p}} \leq \frac{1}{2 \tilde{q}}+\frac{n-1}{4 p} \leq \frac{1}{2 \tilde{q}}+\frac{n-1}{4 \tilde{p}}+\frac{1}{200}<\frac{n-1}{4}
$$

Hence, up to a constant, we can bound the last sum by

$$
\begin{aligned}
& \sum_{k \in \mathbb{Z}} \sum_{l<k} \sup _{(\tilde{q}, \tilde{p}) \in \mathcal{D}^{\#}} 2^{l(s-1)} 2^{n l(1-1 / r)} 2^{2 k\left(1-\frac{1}{2 \tilde{q}}-\frac{n}{2 p}\right)}\left\|f_{k}\right\|_{\mathcal{S}_{k}^{(-1)}}\left\|\tilde{Q}_{k}(g)\right\|_{\mathcal{S}_{k}^{(-1)}} \\
& \lesssim \sum_{k \in \mathbb{Z}} \sum_{j \geq 0} \sup _{(\tilde{q}, \tilde{p}) \in \mathcal{D}^{\#}} 2^{-j\left(\frac{1}{\tilde{q}}+\frac{n}{p}-2\right)}\left\|f_{k}\right\|_{\mathcal{S}_{k}^{(-1)}\left\|\tilde{Q}_{k}(g)\right\|_{\mathcal{S}_{k}^{(-1)}} .}
\end{aligned}
$$


Since $n \geq 4$ and $(\tilde{q}, \tilde{p}) \in \mathcal{D}^{\#}$, we have that $2 \leq 2 \tilde{p} \leq 6$ and hence,

$$
\begin{aligned}
\frac{1}{\tilde{q}}+\frac{n}{p}>\frac{1}{\tilde{q}}+\frac{n}{\tilde{p}} & =2\left(\frac{n-1}{4}\right)^{-}-2 \frac{(n-1)}{4 \tilde{p}}+\frac{2 n}{2 \tilde{p}} \\
& =2\left(\frac{n-1}{4}\right)^{-}+\frac{n+1}{2 \tilde{p}} \\
& >2\left(\frac{n-1}{4}\right)^{-}+\frac{n+1}{6} \\
& >2\left(\frac{3}{4}\right)^{-}+\frac{5}{6}>2^{+} .
\end{aligned}
$$

Thus, we can sum in $j \geq 0$ above; and the desired estimate follows by Cauchy-Schwartz in the sum over $k$.

- We consider (2.12)(ii).

We proceed as follows,

$$
\begin{aligned}
& \sum_{l \in \mathbb{Z}} \sup _{(\tilde{q}, \tilde{p}) \in \mathcal{G}} 2^{-l} 2^{l(1 / \tilde{q}+n / \tilde{p}-1)}\left\|Q_{l}\left(\sum_{m>5} f_{l} \cdot g_{l-m}\right)\right\|_{L_{t}^{\tilde{L}} L_{x}^{\tilde{p}}} \\
& \lesssim \sum_{l \in \mathbb{Z}} \sum_{m>5} \sup _{(\tilde{q}, \tilde{p}) \in \mathcal{E}} 2^{-l} 2^{l(1 / \tilde{q}+n / \tilde{p}-1)}\left\|Q_{l}\left(f_{l} \cdot g_{l-m}\right)\right\|_{L_{t}^{\tilde{q}} L_{x}^{\tilde{p}}} \\
& \lesssim \sum_{l \in \mathbb{Z}} \sum_{m>5} \sup _{(\tilde{q}, \tilde{p}) \in \mathcal{E}} 2^{l(1 / \tilde{q}+n / \tilde{p}-2)}\left\|f_{l}\right\|_{L_{t}^{q_{1}} L_{x}^{p_{1}}\left\|g_{l-m}\right\|_{L_{t}^{q_{2}} L^{p_{2}}}}
\end{aligned}
$$

by Hölder's inequality with $\frac{1}{\tilde{q}}=\frac{1}{q_{1}}+\frac{1}{q_{2}}, \frac{1}{\tilde{\tilde{p}}}=\frac{1}{p_{1}}+\frac{1}{p_{2}}$ and $\left(q_{1}, p_{1}\right) \in \mathcal{A}$, $\left(q_{2}, p_{2}\right) \in \mathcal{C} \subset \mathcal{A}$. Recall also that $\left\|Q_{l}\right\|_{1}=1$.

By the Strichartz inequalities we then have that the sum above is bounded by

$$
\begin{gathered}
\sum_{l \in \mathbb{Z}} \sum_{m>5} \sup _{(\tilde{q}, \tilde{p}) \in \mathcal{E}} 2^{l(1 / \tilde{q}+n / \tilde{p}-2)} 2^{l(2-1 / \tilde{q}-n / \tilde{p})} 2^{-m\left(1-1 / q_{2}-n / p_{2}\right)}\left\|f_{l}\right\|_{\mathcal{S}_{l}^{(-1)}\left\|g_{l-m}\right\|_{\mathcal{S}_{l-m}^{(-1)}}} \\
\lesssim \sum_{m>5} 2^{-m(1 / 100)} \sum_{l \in \mathbb{Z}}\left\|f_{l}\right\|_{\mathcal{S}_{l}^{(-1)}\left\|g_{l-m}\right\|_{\mathcal{S}_{l-m}^{(-1)}}}
\end{gathered}
$$

from where the desired estimates follows by doing first Cauchy Schwartz in the sum over $l \in Z$ and finally summing over $m>5$.

To obtain the desired estimate for the second supremum in the definition of the $\mathcal{S}_{+}^{(-1)}$-norm we need to show a companion estimates to $(2.12)$ (i) and (2.12) (ii).

The high-low estimate for a time derivative is treated in a similar manner to (2.12)(ii). Indeed, the time derivative first introduces a 'loss' of $2^{l}$ and 
then one recoups $2^{l}$ (or even $2^{l-m}$ ) from the estimate for $\left\|\partial_{t} f_{l}\right\|_{L_{t}^{q_{1}} L_{x}^{p_{1}}}$. We omit the details for that part and we concentrate instead on the high-high interaction. To this end, we will show that

$$
\sum_{l} 2^{l(1 / \tilde{q}+n / \tilde{p}-3)} \sum_{k \geq l} \sup _{(\tilde{q}, \tilde{p}) \in D_{t}^{\#}}\left\|Q_{l}\left(\partial_{t} f_{k} \tilde{Q}_{k} g\right)\right\|_{L_{t}^{\tilde{q}} L_{x}^{\tilde{p}}} \lesssim\|f\|_{\mathcal{S}_{k}^{(-1)}}\|g\|_{\mathcal{S}_{k}^{(-1)}}
$$

where $D_{t}^{\#}=\left\{(\tilde{q}, \tilde{p}): \tilde{q} \geq 2, \frac{1}{2 \tilde{q}}+\frac{(n-1)}{4 \tilde{p}}=\frac{n-1}{4}-\right\}$. We note that the case, when the time derivative falls on $\tilde{Q}_{k} g$ is symmetric. By applying the same estimates as in (2.12)(i), one obtains that the sum is bounded by

$$
\sum_{l} \sum_{l \leq k} \sup _{(\tilde{q}, \tilde{p}) \in D_{t}^{\#}} 2^{l(1 / \tilde{q}+n / p-3)} 2^{k} 2^{2 k(1-1 / 2 \tilde{q}-n / 2 p)}\|f\|_{\mathcal{S}_{k}^{(-1)}}\|g\|_{\mathcal{S}_{k}^{(-1)}},
$$

since by definition $\left\|\partial_{t} f_{k}\right\|_{L_{t}^{2 \tilde{q}} L_{x}^{2 p}} \lesssim 2^{k} 2^{k(1-1 / 2 \tilde{q}-n / 2 \tilde{p})}\|f\|_{\mathcal{S}_{k}^{(-1)}}$. Therefore, we need to bound

$$
\sum_{l} \sum_{l \leq k(\tilde{q}, \tilde{p}) \in D_{t}^{\#}} 2^{(l-k)(1 / \tilde{q}+n / \tilde{p}-3)}\|f\|_{\mathcal{S}_{k}^{(-1)}}\|g\|_{\mathcal{S}_{k}^{(-1)}} .
$$

which amounts to verifying $1 / \tilde{q}+n / \tilde{p} \geq 3+$, which is somewhat stronger than what was needed in (2.12)(i). We have

$$
\frac{1}{\tilde{q}}+\frac{n}{p}>\frac{1}{\tilde{q}}+\frac{n}{\tilde{p}}=2\left(\frac{1}{2 \tilde{q}}+\frac{n-1}{4 \tilde{p}}\right)+\frac{n+1}{2 \tilde{p}} \geq \frac{n-1}{2}+\frac{n+1}{2 \tilde{p}}-\frac{1}{50} .
$$

The restriction $\tilde{q} \geq 2$ in the definition of $D_{t}^{\#}$ implies $\tilde{p} \leq(n-1)(n-2)$ and thus

$$
\frac{1}{\tilde{q}}+\frac{n}{p}>\frac{n-1}{2}+\frac{(n+1)(n-2)}{2(n-1)}-\frac{1}{50}>3
$$

for $n \geq 4$. We note that one can do a slightly larger domain of admissible pairs in the time derivative estimates, but $D_{t}^{\#}$ will suffice to close the estimates later on.

Remark. As a consequence of Theorem (2.12) we have that any element in $\mathcal{S}_{+}^{(-1)}$ belongs to both $\mathcal{B}_{1} \subset L_{t}^{1} L_{x}^{\infty}$ and $L_{t}^{2} \dot{B}_{2,2}^{n / 2-1 / 2}$. This will imply, in particular, that the connection 1-form a - whose existence, uniqueness and regularity is established in section 3- belongs to $\mathcal{B}_{1} \subset L_{t}^{1} L_{x}^{\infty}$ and $L_{t}^{2} \dot{B}_{2,2}^{n / 2-1 / 2}$. This is the crucial fact needed to obtain the apriori bounds on the nonlinearity (c.f. Theorem 2.13 below). 
Remark. Although the above embeddings will suffice for our purposes in the present paper; it is interesting to note that in fact, $|\nabla|^{-1}$ maps $\mathcal{S}^{(-1)} \times \mathcal{S}^{(-1)}$ into a slightly larger class of Besov spaces. Namely into $L_{t}^{q} \dot{B}_{\tilde{p}, 2}^{s}$ for any $q \geq 2, s=1 / q+n / \tilde{p}-1$ and $\tilde{p} \geq p$; where $p<2$ is such that $\frac{1}{q}+\frac{n}{p}=\frac{n}{2}+1$.

Theorem 2.13. Let $a \in \mathcal{S}_{+}^{(-1)}$ and $b \in \mathcal{S}^{(-1)}$ then

$$
\left(\sum_{k \in \mathbb{Z}} 2^{2 k(n / 2-1)}\left\|Q_{k}(a \cdot b)\right\|_{L_{t}^{1} L_{x}^{2}}^{2}\right)^{1 / 2} \lesssim\|a\|_{\mathcal{S}_{+}^{(-1)}}\|b\|_{\mathcal{S}^{(-1)}}
$$

Proof. We start as usual by performing a Littlewood-Paley decomposition of $a$ and $b$. We obtain

$$
\begin{aligned}
& \sum_{k \in \mathbb{Z}} 2^{2 k(n / 2-1)}\left\|Q_{k}(a \cdot b)\right\|_{L_{t}^{1} L_{x}^{2}}^{2} \\
& \lesssim \sum_{k \in \mathbb{Z}} 2^{2 k(n / 2-1)}\left\|\sum_{m>5} Q_{k}\left(Q_{k}(a) \cdot Q_{k-m}(b)\right)\right\|_{L_{t}^{1} L_{x}^{2}}^{2} \\
& \quad+\sum_{k \in \mathbb{Z}} 2^{2 k(n / 2-1)}\left\|\sum_{m>5} Q_{k}\left(Q_{k-m}(a) \cdot Q_{k}(b)\right)\right\|_{L_{t}^{1} L_{x}^{2}}^{2} \\
& \quad+\sum_{k \in \mathbb{Z}} 2^{2 k(n / 2-1)}\left\|\sum_{k<l} Q_{k}\left(Q_{l}(a) \cdot Q_{l}(b)\right)\right\|_{L_{t}^{1} L_{x}^{2}}^{2} .
\end{aligned}
$$

Now since $a$ and $b$ belong to different spaces we lose the 'symmetry' and need to consider all three cases separately.

- We consider the first of the three sums above.

$$
\begin{aligned}
& \sum_{k \in \mathbb{Z}} \sum_{m>5} 2^{k(n / 2-1)}\left\|Q_{k}\left(Q_{k}(a) \cdot Q_{k-m}(b)\right)\right\|_{L_{t}^{1} L_{x}^{2}} \\
& \lesssim \sum_{m>5} \sum_{k \in \mathbb{Z}} 2^{k(n / 2-1)}\left\|Q_{k}(a)\right\|_{L_{t}^{2} L_{x}^{2}}\left\|Q_{k-m}(b)\right\|_{L_{t}^{2} L_{x}^{\infty}} \\
& \lesssim \sum_{m>5} 2^{-m / 2} \sum_{k \in \mathbb{Z}} 2^{k(n / 2-1 / 2)}\left\|Q_{k}(a)\right\|_{L_{t}^{2} L_{x}^{2}}\left\|b_{k-m}\right\|_{\mathcal{S}_{k}^{(-1)}}
\end{aligned}
$$

since the pair $(2, \infty)$ is admissible. Note that the pair $(2,2) \in \mathcal{G}$, whence $\left\|Q_{k}(a)\right\|_{L_{t}^{2} L_{x}^{2}} \leq 2^{k(1 / 2-n / 2)}\left\|Q_{k}(a)\right\|_{\mathcal{S}_{k_{+}}(-1)}$-since $1 / 2+n / 2-1=n / 2-1 / 2-$. Finally do Cauchy- Schwartz and the desired estimate follows after summing over $m>5$ last. 
- We consider next the second sum.

$$
\begin{aligned}
& \sum_{k \in \mathbb{Z}} 2^{2 k(n / 2-1)}\left\|\sum_{m>5} Q_{k}\left(Q_{k}(b) \cdot Q_{k-m}(a)\right)\right\|_{L_{t}^{1} L_{x}^{2}}^{2} \\
& \lesssim \sum_{k \in \mathbb{Z}} 2^{2 k(n / 2-1)}\left\|Q_{k}(b)\right\|_{L_{t}^{\infty} L_{x}^{2}}^{2}\left(\sum_{m>5}\left\|Q_{k-m}(a)\right\|_{L_{t}^{1} L_{x}^{\infty}}\right)^{2} \\
& \lesssim\|a\|_{\mathcal{S}_{+}^{(-1)}}^{2} \sum_{k \in \mathbb{Z}}\left\|Q_{k}(b)\right\|_{\mathcal{S}_{k}^{(-1)}}^{2} \\
& \lesssim\|a\|_{\mathcal{S}_{+}^{(-1)}}^{2}\|b\|_{\mathcal{S}^{(-1)}}^{2}
\end{aligned}
$$

- Finally, we consider the third sum.

$$
\begin{aligned}
& \sum_{k \in \mathbb{Z}} \sum_{k<l} 2^{k(n / 2-1)}\left\|Q_{k}\left(Q_{l}(b) \cdot Q_{l}(a)\right)\right\|_{L_{t}^{1} L_{x}^{2}} \\
& \lesssim \sum_{k \in \mathbb{Z}} \sum_{k<l} 2^{k(n / 2-1)}\left\|Q_{l}(b)\right\|_{L_{t}^{\infty} L_{x}^{2}}\left\|Q_{l}(a)\right\|_{L_{t}^{1} L_{x}^{\infty}} \\
& \lesssim \sum_{l \in \mathbb{Z}} \sum_{j \geq 0} 2^{-j(n / 2-1)}\left\|Q_{l}(b)\right\|_{\mathcal{S}_{l}^{(-1)}}\left\|Q_{l}(a)\right\|_{L_{t}^{1} L_{x}^{\infty}} \\
& \lesssim \sum_{l \in \mathbb{Z}}\left\|Q_{l}(b)\right\|_{\mathcal{S}_{l}^{(-1)}}\left\|Q_{l}(a)\right\|_{L_{t}^{1} L_{x}^{\infty}},
\end{aligned}
$$

from where by Cauchy-Schwartz we obtain the desired estimate invoking once again the fact that $a \in \mathcal{B}_{2}$.

\section{Existence, uniqueness and regularity of the connection 1-form in $\mathcal{S}^{(-1)}$.}

Proposition 3.1. Let $b \in \mathcal{S}^{(-1)}$ have sufficiently small norm; then the map

$$
\Phi(w)=|\nabla|^{-1}[w, w]+|\nabla|^{-1}[b, b]
$$

has a unique fixed point $a=\Phi(a) \in \mathcal{S}^{(-1)}$. Moreover, the fixed point $a$ belongs to $L_{t}^{1} L_{x}^{\infty} \cap \dot{B}_{\tilde{p}, 2}^{s}$ for any $\tilde{p} \geq 2$ and $n / \tilde{p}-1 \leq s \leq n / \tilde{p}-1 / 2$.

Proof. Let $b \in \mathcal{S}^{(-1)}$ such that $\|b\|_{\mathcal{S}^{(-1)}}=\epsilon \leq \frac{1}{100 c_{1}}$ where $c_{1}>0$ is the constant from (2.12) such that $\left\||\nabla|^{-1}[\alpha, \beta]\right\|_{\mathcal{S}_{+}^{(-1)}} \leq c_{1}\|\alpha\|\left\|_{\mathcal{S}^{(-1)}}\right\| \beta \|_{\mathcal{S}^{(-1)}}$.

Let $0<r<\epsilon$ and let $B=B_{r}(0)$ be the ball in $\mathcal{S}^{(-1)}$ centered at 0 and radius $r$. Then 
(1) $\Phi: B_{r}(0) \rightarrow B_{r}(0)$

(2) $\left\|\Phi\left(w_{1}\right)-\Phi\left(w_{2}\right)\right\|_{\mathcal{S}^{(-1)}}<\left\|w_{1}-w_{2}\right\|_{\mathcal{S}^{(-1)}}$

To check (1) let $w \in B_{r}(0)$ then

$$
\|\Phi(w)\|_{\mathcal{S}^{(-1)}} \leq c_{1}\|w\|_{\mathcal{S}^{(-1)}}^{2}+c_{1}\|b\|_{\mathcal{S}^{(-1)}}^{2}<r
$$

To check (2) let $w_{1}, w_{2} \in B_{r}(0)$ then

$$
\begin{aligned}
\left\|\Phi\left(w_{1}\right)-\Phi\left(w_{2}\right)\right\|_{\mathcal{S}^{(-1)}} & \leq c_{1}\left\|w_{1}-w_{2}\right\|_{\mathcal{S}^{(-1)}} \max _{i=1,2}\left\{\left\|w_{i}\right\|_{\mathcal{S}^{(-1)}}\right\} \\
& \leq \frac{1}{100}\left\|w_{1}-w_{2}\right\|_{\mathcal{S}^{(-1)}}<\left\|w_{1}-w_{2}\right\|_{\mathcal{S}^{(-1)}}
\end{aligned}
$$

Thus $\Phi$ is a contraction and hence there exists a unique fixed point $a=\Phi(a) \in \mathcal{S}^{(-1)}$ such that

$$
a=|\nabla|^{-1}[a, a]+|\nabla|^{-1}[b, b]
$$

By Lemma 2.12, $|\nabla|^{-1}[\cdot, \cdot] \in L_{t}^{1} L_{x}^{\infty} \cap \dot{B}_{\tilde{p}, 2}^{s}$ for any $\tilde{p} \geq 2$ and $s=1 / \tilde{q}+$ $n / \tilde{p}-1$ with $\tilde{q} \geq 2$. Hence so does $a$.

\section{The Modified Wave Map System.}

In this section we prove that the Cauchy problem for the MWM system derived in Section 2 has a unique global solution in $L^{\infty}\left(\mathbb{R} ; \dot{H}_{x}^{n / 2}\right)$ provided the initial data has sufficiently small $\dot{H}_{x}^{n / 2} \times \dot{H}_{x}^{n / 2-1}$ norm.

Let us denote by $B(a, b)$ the quadratic form equal to any finite linear combination of functions $a \in \mathcal{S}_{+}^{(-1)}$ and $b \in \mathcal{S}^{(-1)}$ of the form $\sum_{\kappa, \ell} c_{\kappa \ell} a_{\kappa} b_{\ell}$ where $a_{\kappa} \in \mathcal{S}_{+}^{(-1)}, b_{\ell} \in \mathcal{S}^{(-1)}$ and $c_{\kappa \ell} \in \mathbb{C}$.

According to our reductions in the previous section, we consider the system of coupled wave equations in $R^{n+1}, n \geq 4$.

$$
\begin{aligned}
\square v & =B(a, b) \\
v(x, 0) & =f(x) \\
v_{t}(x, 0) & =g(x)
\end{aligned}
$$

Lemma 4.2. Let $a \in \mathcal{S}_{+}^{(-1)}$ and $b \in \mathcal{S}^{(-1)}$. Then the solution to the $M W M$ system (4.1) with initial data $(f, g) \in \dot{H}^{n / 2} \times \dot{H}^{n / 2-1}$ satisfies

$$
\|v\|_{\mathcal{S}} \lesssim\|f\|_{\dot{H}^{n / 2}}+\|g\|_{\dot{H}^{n / 2-1}}+\|a\|_{\mathcal{S}_{+}^{(-1)}}\|b\|_{\mathcal{S}^{(-1)}}
$$


Proof. Let us denote by $v_{k}=Q_{k}(v)$. By the Strichartz estimates, we have that

$$
\left\|v_{k}\right\|_{\mathcal{S}_{k}} \lesssim\left\|f_{k}\right\|_{\dot{H}^{n / 2}}+\left\|g_{k}\right\|_{\dot{H}^{n / 2-1}}+2^{k(n / 2-1)}\|B(a, b)\|_{L_{t}^{1} L_{x}^{2}}
$$

from where by Theorem ( 2.13) we have that

$$
\begin{aligned}
\|v\|_{\mathcal{S}} & =\left(\sum_{k \in \mathbb{Z}}\left\|v_{k}\right\|_{\mathcal{S}_{k}}^{2}\right)^{1 / 2} \\
& \lesssim\|f\|_{\dot{H}^{n / 2}}+\|g\|_{\dot{H}^{n / 2-1}}+\left(\sum_{k \in \mathbb{Z}} 2^{2 k(n / 2-1)}\|B(a, b)\|_{L_{t}^{1} L_{x}^{2}}^{2}\right)^{1 / 2} \\
& \lesssim\|f\|_{\dot{H}^{n / 2}}+\|g\|_{\dot{H}^{n / 2-1}}+\|a\|_{\mathcal{S}_{+}^{(-1)}}\|b\|_{\mathcal{S}^{(-1)}}
\end{aligned}
$$

as desired.

Theorem 4.3 (Existence). There exists $\varepsilon>0$ such that whenever the initial data $\|(f, g)\|_{\dot{H}^{n / 2} \times \dot{H}^{n / 2-1}}<\varepsilon$, the system (4.1) has a unique global solution $v \in \mathcal{S}$.

In particular, the solution $v$ belongs both to

$$
\begin{aligned}
& -L^{\infty}\left(\mathbb{R} ; \dot{H}_{x}^{n / 2}\right) \cap L^{2}\left(\mathbb{R} ; \dot{B}_{2 n, 2}^{1}\right) \quad \text { and } \\
& \text { - } W^{1, \infty}\left(\mathbb{R} ; \dot{H}_{x}^{n / 2-1}\right) \cap W^{1,2}\left(\mathbb{R} ; \dot{B}_{2 n, 2}^{0}\right) .
\end{aligned}
$$

Moreover, there is stability; i.e.

$$
\operatorname{ess} \sup _{t}\left\|v_{1}-v_{2}\right\|_{\dot{H}^{n / 2}} \lesssim\left\|\left(f_{1}, g_{1}\right)-\left(f_{2}, g_{2}\right)\right\|_{\dot{H}^{n / 2} \times \dot{H}^{n / 2-1}}
$$

provided the r.h.s. is small enough.

Proof. The proof proceeds by Picard's iteration relying on the a priori estimates as well as the necessary smallness of the data.

Suppose $\|(f, g)\|_{\dot{H}^{n / 2} \times \dot{H}^{n / 2-1}}=\delta$ and let $v_{0}$ be the solution to

$$
\square v_{0}=0 ; \quad v_{0}(0, \cdot)=f \quad \partial_{t} v_{0}(0, \cdot)=g .
$$

By the Strichartz estimates

$$
\left\|v_{0}\right\|_{\mathcal{S}} \leq c_{1}\|(f, g)\|_{\dot{H}^{n / 2} \times \dot{H}^{n / 2-1}}=c_{1} \delta .
$$


Now, $v_{0}=\left(\varphi_{0}, \psi_{0}\right)$ produces $b_{0}=d \varphi_{0}+d i v_{(s p . t)} \psi_{0}$ with $\left\|b_{0}\right\|_{\mathcal{S}^{(-1)}} \leq$ $c_{2}\left\|v_{0}\right\|_{\mathcal{S}} \leq c_{3} \delta$.

Next, the multiplication estimates allow one to perform a fixed point argument to produce $a_{0}$ from $b_{0}$ by solving

$$
a_{0}=|\nabla|^{-1}\left[a_{0}, a_{0}\right]+|\nabla|^{-1}\left[b_{0}, b_{0}\right] .
$$

Moreover, $\left\|a_{0}\right\|_{\mathcal{S}_{+}^{(-1)}} \leq c_{4}\left\|b_{0}\right\|_{\mathcal{S}^{(-1)}}^{2} \leq c_{5} \delta^{2}$

Let $v_{1}$ be the solution of

$$
\square v_{1}=B\left(a_{0}, b_{0}\right) \quad v_{1}(0, \cdot)=f \quad \partial_{t} v_{1}(0, \cdot)=g .
$$

By the a priori estimate,

$$
\left\|v_{1}\right\|_{\mathcal{S}} \leq c_{0}\left(\delta+\left\|a_{0}\right\|_{\mathcal{S}_{+}^{(-1)}}\left\|b_{0}\right\|_{\mathcal{S}^{(-1)}}\right) \leq 2 c_{0} \delta
$$

provided $\delta$ is small enough.

We proceed next by induction to show that for any $j \geq 0,\left\|b_{j}\right\|_{\mathcal{S}} \leq 2 c_{2} c_{o} \delta$, $\left\|a_{j}\right\|_{\mathcal{S}} \leq c_{5} \delta^{2}$ and thus $\left\|v_{j+1}\right\|_{\mathcal{S}} \leq 2 c_{0} \delta$ provided $\delta>0$ is small enough (indep. of $j$ ), where $v_{j+1}$ is the solution to

$$
\square v_{j+1}=B\left(a_{j}, b_{j}\right) \quad v_{j+1}(0, \cdot)=f \quad \partial_{t} v_{j+1}(0, \cdot)=g .
$$

Note that once again by the a priori estimates

$$
\left\|v_{j+1}\right\|_{\mathcal{S}} \leq c_{0}\left(\|(f, g)\|_{\dot{H}^{n / 2} \times \dot{H}^{n / 2-1}}+\left\|a_{j}\right\|_{\mathcal{S}_{+}^{(-1)}}\left\|b_{j}\right\|_{\mathcal{S}^{(-1)}}\right) .
$$

Lastly, for the differences,

$$
\begin{aligned}
\square\left(v_{j+2}-v_{j+1}\right) & =B\left(a_{j+1}, b_{j+1}\right)-B\left(a_{j}, b_{j}\right) \\
& =B\left(a_{j+1}-a_{j}, b_{j+1}\right)+B\left(a_{j}, b_{j+1}-b_{j}\right) \\
v_{j+1}(0, \cdot) & =0 \\
\partial_{t} v_{j+1}(0, \cdot) & =0 .
\end{aligned}
$$

On the other hand note that since

$$
\begin{aligned}
a_{j+1}-a_{j}= & |\nabla|^{-1}\left[a_{j+1}-a_{j}, a_{j+1}\right]+|\nabla|^{-1}\left[a_{j}, a_{j+1}-a_{j}\right] \\
& +|\nabla|^{-1}\left[b_{j+1}-b_{j}, b_{j+1}\right]+|\nabla|^{-1}\left[b_{j}, b_{j+1}-b_{j}\right] \\
& \left\|b_{j+1}-b_{j}\right\|_{\mathcal{S}(-1)} \leq c_{2}\left\|v_{j+1}-v_{j}\right\|_{\mathcal{S}}
\end{aligned}
$$


and

$$
\left\|a_{j+1}\right\|_{\mathcal{S}^{(-1)}},\left\|a_{j}\right\|_{\mathcal{S}^{(-1)}},\left\|b_{j+1}\right\|_{\mathcal{S}^{(-1)}},\left\|b_{j}\right\|_{\mathcal{S}^{(-1)}} \leq c \delta
$$

we have that

$$
\begin{aligned}
& \left\|a_{j+1}-a_{j}\right\|_{\mathcal{S}_{+}^{(-1)}} \\
& \leq c\left\|a_{j+1}\right\|_{\mathcal{S}^{(-1)}}\left\|a_{j+1}-a_{j}\right\|_{\mathcal{S}^{(-1)}}+\left\|a_{j}\right\|_{\mathcal{S}^{(-1)}}\left\|a_{j+1}-a_{j}\right\|_{\mathcal{S}^{(-1)}} \\
& \quad+\left\|b_{j+1}\right\|_{\mathcal{S}^{(-1)}}\left\|b_{j+1}-b_{j}\right\|_{\mathcal{S}^{(-1)}}+\left\|b_{j}\right\|_{\mathcal{S}^{(-1)}}\left\|b_{j+1}-b_{j}\right\|_{\mathcal{S}^{(-1)}} \\
& \leq c \delta\left\|a_{j+1}-a_{j}\right\|_{\mathcal{S}^{(-1)}}+c^{\prime} \delta\left\|b_{j+1}-b_{j}\right\|_{\mathcal{S}^{(-1)}} .
\end{aligned}
$$

Hence,

$$
\left\|a_{j+1}-a_{j}\right\|_{\mathcal{S}_{+}^{(-1)}} \leq c \delta\left\|b_{j+1}-b_{j}\right\|_{\mathcal{S}^{(-1)}} \leq c \delta\left\|v_{j+1}-v_{j}\right\|_{\mathcal{S}}
$$

All in all we then have that

$$
\begin{aligned}
& \left\|v_{j+2}-v_{j+1}\right\|_{\mathcal{S}} \\
& \leq c\left(\left\|a_{j+1}-a_{j}\right\|_{\mathcal{S}_{+}^{(-1)}}\left\|b_{j+1}\right\|_{\mathcal{S}^{(-1)}}+\left\|a_{j+1}\right\|_{\mathcal{S}^{(-1)}}\left\|b_{j+1}-b_{j}\right\|_{\mathcal{S}^{(-1)}}\right) \\
& \leq c \delta^{2}\left\|v_{j+1}-v_{j}\right\|_{\mathcal{S}}
\end{aligned}
$$

Finally, by choosing $\delta$ small enough we have that

$$
\left\|v_{j+2}-v_{j+1}\right\|_{\mathcal{S}} \leq \frac{1}{2}\left\|v_{j+1}-v_{j}\right\|_{\mathcal{S}}
$$

Hence $v_{j}$ is Cauchy in $\mathcal{S}$, thus establishing existence and uniqueness. For the stability result one proceeds in the same fashion as in the proof of being Cauchy; thus concluding the proof of the theorem.

The theorem above gives uniqueness solely in $\mathcal{S}$ which is not enough to claim the solution to the MWM system came from a wave map. Thus we proceed next to prove a stronger uniqueness result which will indeed suffice in section 5 to return to the wave map.

Theorem 4.4 (Uniqueness). Suppose $\left(v_{1}, a_{1}\right)$ and $\left(v_{2}, a_{2}\right)$ are two solutions to

$$
\begin{aligned}
& \square v+B(a, d v)=0 \\
& \Delta a+\operatorname{div} B(a, a)+\operatorname{div} B(d v, d v)=0
\end{aligned}
$$


such that $d v_{j}=b_{j}$, for $j=1,2$ are small in $L_{t}^{\infty} L_{x}^{n}$. Suppose that $d v_{j}=b_{j} \in$ $L_{t}^{2} L_{x}^{2 n}$ for $j=1,2$. Assume in addition that $a_{1}=a_{1}\left(v_{1}\right) \in L_{t}^{1} L_{x}^{\infty}$. Then $v_{1}=v_{2}$.

Remark The smallness of $d v_{j}$ in $L_{t}^{\infty} L_{x}^{n}$ is the necessary condition to solve the 'gauged' equation. Indeed this condition corresponds to the smallness of the $L_{t}^{\infty} L_{x}^{n}$ of $d s$ which is the necessary condition in Corollary 1.3 guaranteeing the existence of a 'good gauge'. Also note that it is not necessary for $a_{2}=a_{2}\left(v_{2}\right)$ to be in $L_{t}^{1} L_{x}^{\infty}$.

Proof. The proof follows the scheme devised by Shatah-Struwe to establish uniqueness [16], [15]. Let us denote

$$
\begin{aligned}
\delta w & =v_{1}-v_{2} \\
\delta a & =a_{1}-a_{2} \\
\delta b & =d v_{1}-d v_{2},
\end{aligned}
$$

and so on. Then,

$$
\begin{gathered}
\square \delta w=B\left(a_{1}, \delta b\right)+B\left(\delta a, b_{2}\right) \\
\int\left\langle\frac{\partial}{\partial t} \delta w, \square \delta w\right\rangle d x=\frac{1}{2} \frac{\partial}{\partial t} E^{2}, \quad \text { where } \\
E^{2}=\int|d(\delta w)|^{2}+\left|\frac{\partial(\delta w)}{\partial t}\right|^{2} d x=\int|\delta b|^{2} d x .
\end{gathered}
$$

Then,

$$
\frac{1}{2} \frac{\partial}{\partial t} E^{2} \leq\left\|a_{1}\right\|_{t, L_{x}^{\infty}} E^{2}(t)+E(t)\|\delta a\|_{t, L_{x}^{\frac{2 n}{n-1}}}\|b\|_{t, L_{x}^{2 n}}
$$

Integrating over $t$ we then obtain that

$$
\begin{aligned}
E^{2}(t) \leq & \max _{\tau \leq t} E^{2}(\tau) \int_{0}^{\tau}\left\|a_{1}\right\|_{t, L_{x}^{\infty}} d t \\
& +\max _{\tau \leq t} E(\tau)\|\delta a\|_{L_{(0, t)}^{2}{ }_{x}^{\frac{2 n}{n-1}}\|b\|_{L_{(0, t)}^{2}}^{2} L_{x}^{2 n}}
\end{aligned}
$$

where $L_{(0, t)}^{2}$ means the $L^{2}$ norm on the time interval $(0, t)$. Now,

$$
\Delta \delta a+\operatorname{div} B\left(\delta a, a_{1}+a_{2}\right)+\operatorname{div} B\left(\delta b, b_{1}, b_{2}\right)=0
$$

Hence,

$$
\begin{aligned}
\|\delta a\|_{\left(t, W_{x}^{1,2 n / n+1}\right)} \leq & \|\delta a\|_{\left(t, L_{x}^{2 n / n-1}\right)}\left(\left\|a_{1}\right\|_{\left(t, L_{x}^{n}\right)}+\left\|a_{2}\right\|_{\left(t, L_{x}^{n}\right)}\right) \\
& +\|\delta b\|_{\left(t, L_{x}^{2}\right)}\left(\left\|b_{1}\right\|_{\left(t, L_{x}^{2 n}\right)}+\left\|b_{2}\right\|_{\left(t, L_{x}^{2 n}\right)}\right)
\end{aligned}
$$


On the other hand, $\left\|a_{j}\right\|_{W^{1, n / 2}} \leq\left\|a_{j}\right\|_{L^{n}}^{2}+\left\|b_{j}\right\|_{L^{n}}^{2}$ and $\left\|b_{j}\right\|_{L^{n}}^{2}$ is small for each $t$. Moreover, by Sobolev embedding $\left\|a_{j}\right\|_{L^{n}} \leq c(n)\left\|a_{j}\right\|_{\dot{W}^{1, n / 2}}$; hence (for example by a fixed point argument in $L^{n}$ similar to Lemma 3.1) we have that $\left\|a_{j}\right\|_{L^{n}}$ is also small for each fixed $t$.

All in all, from (4.6) we have that,

$$
\|\delta a\|_{t, L_{x}^{\frac{2 n}{n-1}}} \leq \tilde{c}(n)\|\delta a\|_{t, L_{x}^{\frac{2 n}{n+1}}} \leq \tilde{c}(n) E(t)\left(\left\|b_{1}\right\|_{\left(t, L_{x}^{2 n}\right)}+\left\|b_{2}\right\|_{\left(t, L_{x}^{2 n}\right)}\right) .
$$

Integrate $\tau \leq t$ to get,

$$
\|\delta a\|_{L_{(0, t)}^{2}, L_{x}^{\frac{2 n}{n-1}}} \leq \tilde{c}(n) \max _{\tau \leq t} E(\tau)\left(\left\|b_{1}\right\|_{L_{(0, t)}^{2}, L_{x}^{2 n}}+\left\|b_{2}\right\|_{L_{(0, t)}^{2}, L_{x}^{2 n}}\right) .
$$

Sticking this estimate back in (4.5) we obtain

$$
E^{2}(t) \leq \max _{\tau \leq t} E^{2}(\tau)\left(\left\|a_{1}\right\|_{L_{(0, t)}^{1} L_{x}^{\infty}}+\tilde{c}(n)\left(\left\|b_{1}\right\|_{L_{(0, t)}^{2}, L_{x}^{2 n}}+\left\|b_{2}\right\|_{L_{(0, t)}^{2}, L_{x}^{2 n}}\right)^{2}\right) .
$$

Since $E(0)=0$, we must then have that $E(t)=0$.

By differentiating the MWM system (4.1) and observing that the resulting nonlinearity has the same bilinear structure -for which the main multiplication estimates hold- the following regularity result follows.

Theorem 4.7 (Higher Regularity). Suppose the initial data $(f, g)$ to (4.1) is in $H^{n / 2+1} \times H^{n / 2}$ and has sufficiently small $\dot{H}^{n / 2} \times \dot{H}^{n / 2-1}$ norm. Then the solution $v$ to the Cauchy problem (4.1) with initial data $(f, g)$ can be continued in $H^{n / 2+1} \times H^{n / 2}$ globally in time. Furthermore, we have the global bounds

$$
\|v\|_{L_{t}^{\infty}\left(\mathbb{R} ; \dot{H}_{x}^{n / 2+1}\right)} \lesssim\|(f, g)\|_{\dot{H}_{x}^{n / 2+1} \times \dot{H}_{x}^{n / 2}}
$$

Proof. Assume for simplicity that the data is infinitely smooth. The constants in our estimates will depend only on the relevant smoothness assumptions in the theorem.

Differentiate (4.1) to get

$$
\begin{aligned}
\square w & =B(\partial a, b)+B(a, \partial b) \\
w(x, 0) & =\partial f(x) \\
w_{t}(x, 0) & =\partial g(x),
\end{aligned}
$$


where $w=\partial v$ ( $\partial$ may signify any of $\partial_{j}$ ). Recall also that $a$ is a (unique) fixed point for $\Phi$ and therefore its derivative will satisfy

$$
\partial a=|\nabla|^{-1}[\partial a, a]+|\nabla|^{-1}[a, \partial a]+|\nabla|^{-1}[\partial b, b]+|\nabla|^{-1}[b, \partial b] .
$$

Estimating both sides in $\|\cdot\|_{\mathcal{S}_{+}^{-1}}$, together with the main multiplication estimate and $\|\cdot\|_{\mathcal{S}_{+}^{-1}} \leq\|\cdot\|_{\mathcal{S}^{-1}}$, yields

$$
\|\partial a\|_{\mathcal{S}_{+}^{-1}} \lesssim\|\partial a\|_{\mathcal{S}_{+}^{-1}}\|a\|_{\mathcal{S}^{-1}}+\|\partial b\|_{\mathcal{S}^{-1}}\|b\|_{\mathcal{S}^{-1}}
$$

Recall from the Picard iteration method, that since $\|(f, g)\|_{\dot{H}^{n / 2} \times \dot{H}^{n / 2-1}}$ is small, we have $\|a\|_{\mathcal{S}_{+}^{-1}}$ and $\|b\|_{\mathcal{S}^{-1}}$ small as well. By the usual hiding argument, one deduces

$$
\|\partial a\|_{\mathcal{S}_{+}^{-1}} \lesssim \frac{\|\partial b\|_{\mathcal{S}^{-1}}\|b\|_{\mathcal{S}^{-1}}}{1-\|a\|_{\mathcal{S}^{-1}}} \leq\|b\|_{\mathcal{S}}\|b\|_{\mathcal{S}^{-1}}
$$

thus placing the nonlinearity $B(\partial a, b)$ in the form $\mathcal{S}_{+}^{-1} \cdot \mathcal{S}^{-1}$ as in Lemma 4.2. Since $b \sim \partial v$, the same holds for the other nonlinearity associated with the derivated equation, namely $B(a, \partial b)$. An application of the Strichartz estimates and Lemma 4.2 yields

$$
\begin{aligned}
\|w\|_{\mathcal{S}} & \lesssim\|\partial f\|_{\dot{H}^{n / 2}}+\|\partial g\|_{\dot{H}^{n / 2-1}}+\|b\|_{\mathcal{S}^{-1}}^{2}\|b\|_{\mathcal{S}} \\
& \lesssim\|(f, g)\|_{H^{n / 2+1} \times H^{n / 2}}+o\left(\|(f, g)\|_{\dot{H}^{n / 2} \times \dot{H}^{n / 2}}^{2}\right)\|w\|_{\mathcal{S}}
\end{aligned}
$$

The result follows, since $\|v\|_{L_{t}^{\infty} H_{x}^{n / 2+1}} \lesssim\|w\|_{\mathcal{S}}$.

\section{The Return to the Map.}

The well-posedness results on the modified wave map apply to a larger class of formal solutions $(a, b)$ to the equation than those which come from wave maps. Our method of using the results on the modified wave map equation to show existence of wave maps is similar to the idea we used for Schrödinger maps [14] and not very different from the technique used by Shatah-Struwe [15]. The translation depends on the compactness of $M$ (or certain bounds on the isometric Nash embedding of a non-compact $M$ in an Euclidean space). The proofs are very simple for the Lie group case because of the natural parallel structure; and the compact symmetric space case (e.g. $\mathbb{S}^{m}$ ) is a special case due to the totally geodesic embedding $G / K \subseteq G$. Since we have estimates only for this case, we restrict to this case; although the theorems below are true in general. 
Theorem 5.1. Let $n \geq 3$. If $\left(s, s^{-1} \frac{\partial s}{\partial t}\right) \in \dot{H}^{n / 2} \times \dot{H}^{n / 2-1}$ are sufficiently small initial data for a wave map into a compact Lie group $G$, then there exists a gauge transformation $g \in \dot{H}^{n / 2}$ and a formal derivative $\frac{\partial g}{\partial t} \in \dot{H}^{n / 2-1}$, such that the initial data

$$
b=\frac{1}{2} g\left(s^{-1} d s\right) g^{-1}
$$

are small in $\dot{H}^{n / 2-1}$. Furthermore, if

$$
a=-d g g^{-1}+\frac{1}{2} g\left(s^{-1} d s\right) g^{-1},
$$

then $a$ satisfies $\sum_{j=1}^{n} \frac{\partial a_{j}}{\partial x^{j}}=0$ and is small in $\dot{W}^{n / 2,2 n /(n+2)} \subset \dot{H}^{n / 2-1}$

Proof. Note that the pull-back connection in the frame of left pull-back to the Lie algrebra is $d+\frac{1}{2} s^{-1} d s$. The curvature is $\frac{1}{4}\left[s^{-1} d s, s^{-1} d s\right]$, which will be small in $L^{n / 2}$ since $\dot{H}^{n / 2-1} \subset L^{n}$. We can then apply Theorem (1.1) (or actually the first step in a time-slice of the proof) to get a good gauge. Since

$$
\sum_{j=1}^{n} \frac{\partial}{\partial x^{j}}\left[\left(d g g^{-1}\right)-g\left(\frac{s^{-1} d s}{2}\right) g-1\right]=0,
$$

a standard regularity theorem will give $g$ to be as smooth as $s$. Here we use heavily the fact that $g$ is bounded. Then $b=g\left(s^{-1} d s\right) g^{-1}$ has components which are small in $\dot{H}^{n / 2-1}$. Since $a=-d g g^{-1}+g\left(\frac{s^{-1} d s}{2}\right) g^{-1}$ has $\sum_{j=1}^{n} \frac{\partial a_{j}}{\partial x^{j}}=$ 0 on the time-slice $t=0 ; a \in \dot{W}^{1, n / 2}$ is small. A standard regularity theorem applied to the equation

$$
\Delta a_{j}+\sum_{k=1}^{n} \frac{\partial}{\partial x^{k}}\left[a_{k}, a_{j}\right]+\sum_{k=1}^{n} \frac{\partial}{\partial x^{k}}\left[b_{k}, b_{j}\right]=0
$$

gives $a_{j} \in \dot{W}^{n / 2,2 n /(n+2)}$ small and bounded by $\|b\|_{\dot{H}^{n / 2-1}}$.

The time derivative $\frac{\partial g}{\partial t}$ is chosen so that if

$$
\begin{aligned}
a_{0} & =-\frac{\partial g}{\partial t} g^{-1}+\frac{1}{2} g^{-1} s^{-1} \frac{\partial s}{\partial t} g \\
0 & =\Delta a_{0}+\sum_{j=1}^{n} \frac{\partial}{\partial x^{j}}\left(\left[a_{j}, a_{0}\right]+\left[b_{j}, b_{0}\right]\right)
\end{aligned}
$$


and $a_{0}$ will also be small in $\dot{W}^{n / 2,2 n /(n+2)}$. This implies $g^{-1} \frac{\partial g}{\partial t}$ is small in $\dot{H}^{n / 2-1}$ as claimed.

The estimates follow from standard composition and multiplication theorems, and elliptic regularity. The needed multiplication theorems are less straightforward for the fractional derivatives needed in odd dimensions, but are extended to the fractional derivatives by interpolation between integral derivatives.

Theorem 5.2. Let $\left(s, s^{-1} \frac{\partial s}{\partial t}\right) \in \dot{H}^{n / 2} \times \dot{H}^{n / 2-1}$ be initial data for a wave map into a compact group. If $s$ is sufficiently close to the identity in $\dot{H}^{n / 2}$, then there exist approximations $\left(s_{\alpha}, \nu_{\alpha}\right)$ in $C_{i d}^{\infty} \times C^{\infty}$ such that

$$
\left(s_{\alpha}, \nu_{\alpha}\right) \rightarrow\left(s, s^{-1} \frac{\partial s}{\partial t}\right) \quad \text { in } \quad \dot{H}^{n / 2} \times \dot{H}^{n / 2-1} .
$$

By $C_{i d}^{\infty}$ we have denoted the space of $C^{\infty}$ maps which are the identity at infinity.

Proof. Let $s \in G \subset \mathbb{R}^{\ell} \times \mathbb{R}^{\ell}$, and $\nu=s^{-1} \frac{\partial s}{\partial t} \in \mathfrak{G}$, which is a vector space. The standard approximation method is to convolve

$$
\nu_{\alpha}(x)=\int \nu\left(x+2^{-\alpha} y\right) \varphi(y) d y=\int \nu\left(x+y^{\prime}\right) \varphi_{\alpha}\left(y^{\prime}\right) d y^{\prime}=J_{\alpha}(\nu)(x)
$$

where $\varphi_{\alpha}\left(y^{\prime}\right)=2^{n \alpha} \varphi\left(2^{\alpha} y^{\prime}\right)$ and $\varphi$ is a smooth bump function with compact support such that $\int \varphi=1$. Since $\nu=s^{-1} \frac{\partial s}{\partial t}$ is in the Lie algebra, this makes sense. The approximation for $s$ is more subtle. Let $\mathbb{P}: \mathcal{U}(G) \rightarrow G$ be the projection operator of a neighborhood of $G$ onto the nearest point in $G$. We define

$$
s_{\alpha}=\mathbb{P}\left(J_{\alpha}(s)\right)
$$

This is well define in the case that

$$
\int_{|x-y| \leq r}|d s| \leq r^{-n+1} \epsilon
$$

for all small $r>0$ and $\epsilon>0$ sufficiently small (depending on the diameter of the neighboorhood $\mathcal{U}(G)$ ). If $s \in \dot{H}^{n / 2}$ is sufficiently small, this will be true. Then, the result that $s_{\alpha} \rightarrow s$ in $\dot{H}^{n / 2}$ follows by applying the regularity or density result of $\mathrm{F}$. Bethuel of smooth maps between certain manifolds in Sobolev spaces ([2]; c.f. in [1]). 
Theorem 5.3. Let $s:[0, T] \times \mathbb{R}^{n} \rightarrow G$ be a wave map in a time interval $[0, T]$ such that $d s \in L_{t}^{\infty} L_{x}^{n} \cap L_{t}^{2} L_{x}^{2 n}$. Assume the initial data is in $\dot{H}^{n / 2} \times$ $\dot{H}^{n / 2-1}$ and has sufficiently small norm. Then $s$ is a gauge transformation of a modified wave map, and $s \in \dot{H}^{n / 2} \times \dot{H}^{n / 2-1}$ remains small in $[0, T]$. Moreover, if the initial data is in $\dot{H}^{n / 2+1} \times \dot{H}^{n / 2}$, then $s \in L_{t}^{\infty} \dot{H}^{n / 2+1}$ and $\frac{\partial s}{\partial t} \in L_{t}^{\infty} \dot{H}^{n / 2}$ for the time the solution exists.

Proof. Since solutions of the wave map are local, we can assume without loss of generality that its $L_{t}^{\infty} L_{x}^{n}$ norm is small for the time interval of existence ( $a$ posteriori this will be true anyway ). Make a gauge transformation to a modified wave map. The gauged modified wave map lies in the regime of our uniqueness theorem (4.4). Therefore, it coincides with the solution we have found (the constructed solution satisfies $a \in L_{t}^{1} L_{x}^{\infty}$ ). Hence it is a gauge transformation of a solution in $\mathcal{S}$. The regularity theorem (4.7) implies the second statement.

We define next $\tilde{\mathcal{S}}^{(-1)}$ as the natural mixed Lebesgue-Sobolev norm space $\mathcal{S}^{(-1)}$ lies in. More precisely,

Definition 5.4. Let $\tilde{\mathcal{S}}^{(-1)}$ be the space of functions on $\mathbb{R} \times \mathbb{R}^{n}$ whose norm is given by

$$
\|\phi\|_{\tilde{\mathcal{S}}^{(-1)}}:=\sup _{\left\{(q, p, s): q, p \geq 2 \frac{1}{q}+\frac{n}{p}-1=s\right\}}\|\phi\|_{L_{t}^{q} \dot{W}^{s, p}} .
$$

Corollary 5.5. Suppose $s:[0, T] \times \mathbb{R}^{n} \rightarrow G$ is a wave map with $d s \in$ $L_{t}^{\infty} L_{x}^{n} \cap L_{t}^{2} L_{x}^{2 n}$. Suppose, in addition, the data at any point of time is small in $\dot{H}^{n / 2} \times \dot{H}^{n / 2-1}$. Then $s$ exists for all time and $d s \in \tilde{\mathcal{S}}^{(-1)}$.

Proof. The gauge transformation of this map coincides with the MWM we have found. Moreover, if $a b, \in \mathcal{S}^{(-1)}$, then solution $g_{ \pm}$of

$$
d g+a g \pm b g=0
$$

exist -since the curvature of $d+a \pm b$ is zero, we can apply Theorem 1.1 - and a standard regularity argument shows that $d g_{ \pm} \in \tilde{\mathcal{S}}^{(-1)}$. Then $s=g_{+} g_{-}^{-1}$ has the same property.

Theorem 5.6. If $(s, \nu) \in H^{n / 2+1} \times H^{n / 2}$ are initial data for a wave map and $(s, \nu) \in \dot{H}^{n / 2} \times \dot{H}^{n / 2-1}$ has small enough norm, then there exists a unique global solution with $d^{2} s \in \tilde{\mathcal{S}}^{(-1)}$. 
Proof. Local existence theorems for data in $H^{n / 2+1} \times H^{n / 2}$ are available ([8] [12]). By theorem 5.3, the norm of $\left(s, s^{-1} \frac{\partial s}{\partial t}\right) \in L_{t}^{\infty} H_{x}^{n / 2+1} \times L_{t}^{\infty} H_{x}^{n / 2}$ remains bounded. Hence the local existence theorems can be used to extend the solution intervals to obtain a unique global solution.

Theorem 5.7. Let $(s, \nu) \in \dot{H}^{n / 2} \times \dot{H}^{n / 2-1}$ be small data for a wave map into a compact group or symmetric space. Then there exists a unique global solution, which is a gauge transformation of a solution to the modified wave equation in $\mathcal{S}$ and hence $d s \in \tilde{\mathcal{S}}^{(-1)}$.

Proof. Approximate $(s, \nu)$ by smooth data $\left(s_{\alpha}, \nu_{\alpha}\right)$. Then there exist global solutions to the wave map problem with initial data $\left(s_{\alpha}, \nu_{\alpha}\right)$. These are gauge transformations of solutions of the modified wave map problem. Choose a weak limit. This limit must have a gauge transformation coinciding with one of our constructed solutions to the modified wave map problem. But since this solution is a weak limit of solutions satisfying $d a+[a, a]+[b, b]=0$, this modified wave map has properties of the complete wave map and can be gauged back (using theorem (1.4) since the curvature of $d+a \pm b$ is zero).

\section{References.}

[1] F. Bethuel. Approximations in trace spaces defined between mainifolds, Nonlinear Analysis, 24, no. 1 (1995), 121-130.

[2] F. Bethuel, The approximation problem for Sobolev maps between two manifolds, Acta Math. 167, no. 3-4 (1991), 153-206.

[3] D. Christodoulou and S. Tahvildar-Zadeh, On the regularity of spherically symmetric wave map, Comm. Pure Appl. Math 46 (1993), 10411091.

[4] M. Grillakis, A priori estimates and regularity for nonlinear waves, Proceedings of the International Congress of Mathematicians, Zürich, 1, 2 (1994), 1187-1194.

[5] M. Grillakis, Classical solutions for the equivariant wave map in $1+2$ dimensions, Indiana Univ. Math. J., to appear.

[6] M. Keel and T. Tao, Endpoint Strichartz estimates Amer. Math. J., 120 (1998), 955-980. 
[7] M. Keel and T. Tao, Local and global well-posedness of wave maps in $R^{1+1}$ for rough data, IMRN, 21 (1998), 1117-1156.

[8] S. Klainerman and M. Machedon, Space-time estimates for null forms and the local existence, Comm. Pure Appl. Math., 46 no. 9 (1993), 1221-1268.

[9] S. Klainerman and M. Machedon, Smoothing estimates for null forms and applications, Duke Math J., 81 (1995), 99-133.

[10] S. Klainerman and M. Machedon, On the optimal local regularity for gauge fields theories, Diff. and Integral Eqs., 10 (1997), 1019-1030.

[11] S. Klainerman and I. Rodnianski, On the global regularity of wave maps in the critical Sobolev norm, IMRN, 13 (2001), 655-677.

[12] S. Klainerman and S. Selberg, Remarks on the optimal regularity for equations of wave maps type, Comm. PDE, 22 (1997), 901-918.

[13] S. Klainerman and D. Tataru, On the optimal local regularity for YangMills equations in $\mathbb{R}^{4+1}$, J. Amer. Math.Soc., 12 (1999), 93-116.

[14] A. Nahmod, A. Stefanov and K. Uhlenbeck, On Schrödinger maps, to appear in CPAM (2002).

[15] J. Shatah and M. Struwe, The Cauchy problem for wave maps, to appear in IMRN (2002).

[16] J. Shatah and M. Struwe, Geometric wave equations, Courant Lecture Notes in Mathematics 2, 1998.

[17] J. Shatah and S. Tahvildar-Zadeh, On the Cauchy problem for equivariant wave maps, Comm. Pure Appl. Math., 47 (1994), 719-753.

[18] J. Shatah and S. Tahvildar-Zadeh, On the stability of stationary wave maps, Comm. Math. Phys., 185, no. 1 (1997), 231-256.

[19] M. Struwe, Equivariant wave maps in two space dimensions, preprint (2000).

[20] M. Struwe, Regularity results for harmonic maps of Minkowski space, Nematics, Coron et al., eds. NATO ASI SER. C 332 (1991), 357-369.

[21] M. Struwe, Geometric Evolution Problems, IAS/Park City Math. Series 2 (1996), 259-339. 
[22] T. Tao, Global regularity of wave maps I. Small critical Sobolev norm in high dimension, IMRN 7 (2001), 299-328.

[23] T. Tao, Global regularity of wave maps II. Small energy in two dimensions, Comm. Math. Phys. to appear.

[24] D. Tataru, Local and global results for wave maps I, Comm. in PDE 23 (1998), 1781-1793.

[25] D. Tataru, On global existence and scattering for the wave maps equation, Amer. J. Math (to appear).

[26] K. Uhlenbeck, Connections with $L^{p}$ bounds on curvature, Comm. Math. Phys. 83 (1982), 31-42.

Department of Mathematics and Statistics

UNIVERSITY OF MASSACHUSETTS

AMHERST, MA 01003-4515

E-mail address: nahmod@math.umass.edu

Department of Mathematics and Statistics

UNIVERSITY OF MASSACHUSETTS

AMHERST, MA 01003-4515

E-mail address: stefan@math.umass.edu

Department of Mathematics

The University of TeXas at Austin

Austin, TX 78712-1082

E-mail address: uhlen@math.utexas.edu

Received November 19, 2001. 